\title{
Beacons for Supporting Lunar Landing Navigation
}

\author{
Stephan Theil • Leonardo Bora
}

Received: date / Accepted: date

\begin{abstract}
Current and future planetary exploration missions involve a landing on the target celestial body. Almost all of these landing missions are currently relying on a combination of inertial and optical sensor measurements to determine the current flight state with respect to the target body and the desired landing site. As soon as an infrastructure at the landing site exists, the requirements as well as conditions change for vehicles landing close to this existing infrastructure. This paper investigates the options for ground based infrastructure supporting the on-board navigation system and analyzes the impact on the achievable navigation accuracy. For that purpose the paper starts with an existing navigation architecture based on optical navigation and extends it with measurements to support navigation with ground infrastructure. A scenario of lunar landing is simulated and the provided functions of the ground infrastructure as well as the location with respect to the landing site are evaluated. The results are analyzed and discussed.
\end{abstract}

Keywords lunar landing · autonomous navigation · beacon navigation

\section{Introduction}

Since the beginning of human space exploration, safe and soft landing on a celestial body (planet, moon, asteroid) has been a central objective. Starting

\author{
S. Theil \\ German Aerospace Center (DLR) \\ Institute of Space Systems, GNC Systems Department \\ Tel.: +49-421-24420-1113 \\ Fax: +49-421-24420-1120 \\ E-mail: stephan.theil@dlr.de \\ L. Bora \\ AIRBUS Defence \& Space Ltd \\ Tel.: +44-1438-77-4186 \\ E-mail: leonardo.bora@airbus.com
}


from the first landings on the Moon, which had generally a precision above 1 $\mathrm{km}$, today improvements in the navigation architecture and filters have made it possible to improve the final accuracy and increase the safety.

The more exploration missions land on a planet the more resources on the ground can be made available for further missions. The potentially first implementation of co-located ground infrastructure is probably a robotic lunar base which will be followed by a human base. For both some studies have been done in the past and are still ongoing (e.g. [27]). The scenario of a robotic or manned lunar base is used as a motivation to investigate the impact of ground infrastructure to the navigation system of later arriving landing and potentially also departing vehicles.

The paper starts in section 2 with the state-of-the-art navigation system architecture for planetary landers and introduces possible measurements from ground based infrastructure to support navigation. From this an extended architecture is generated which is based on the usage of ground beacons providing radio frequency $(\mathrm{RF})$ based measurements.

Section 3 introduces a lunar landing scenario as a reference mission for the analysis of the effect of the ground beacons. Current navigation requirements for landing vehicles are established and an evaluation function is defined.

In section 4 simulation results are presented first for a baseline navigation system without ground support. Then the results for two different beacon configurations are presented. For each configuration a grid of potential positions with respect to the target landing site is analyzed and evaluated with the function defined in section 3. Finally, the results from all test cases are discussed and a recommendation for a beacon location is given.

\section{Navigation System Architecture}

\subsection{Independent Architecture of Autonomous Navigation System}

The baseline navigation system architecture is based on the project SINPLEX (Small Integrated Navigator for PLanetary EXploration) [25]. Figure 1 shows an overview of the general architecture. The inputs used by the navigation system are sensor measurements and image processing results from:

- an Inertial Measurement Unit (IMU),

- a star tracker (ST),

- a laser altimeter (LA),

- a feature tracking algorithm (FT) providing feauture positions in the camera frame, and

- a crater navigation algorithm $(\mathrm{CN})$ providing absolute position measurements[18],

where the results of feature tracking and crater navigation are obtained from processing images taken by a navigation camera.

The navigation filter is a discrete delayed error-state EKF (eEKF), which copes with the fact that measurements in the real system are not instantly 

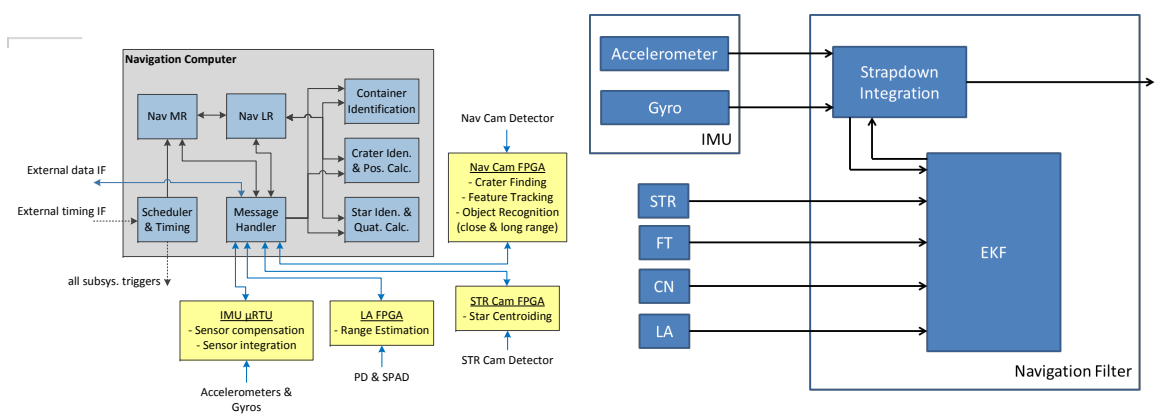

Fig. 1 Functional block diagram (left) and software architectur (right) of the SINPLEX navigation system.

available but with delays. For SINPLEX a delayed eEKF developed in [24] was used which is able to handle delays in an efficient way. Although the delayed eEKF exist in a real-time implementation in this analysis a preparatory version of the navigation software without the delay for the updates has been used. The states necessary for the strapdown navigation are position and velocity in Moon Centered Moon Fixed Frame $(\mathrm{MCMF})^{1}$ frame, attitude error angles and accelerometer and gyro biases and scale factors. There are then additional states necessary for the terrain relative navigation, which are feature positions needed to build the terrain model. The terrain model is estimated through the solution of the SLAM problem as proposed in [10].

The state vector is

$$
\mathbf{x}=\left\{\begin{array}{c}
\mathbf{r}^{M C M F} \\
\mathbf{v}^{M C M F} \\
\boldsymbol{\theta}^{B} \\
\mathbf{b}_{a}^{B} \\
\mathbf{s}_{a}^{B} \\
\mathbf{b}_{g}^{B} \\
\mathbf{s}_{g}^{B} \\
\boldsymbol{\xi}_{i}^{M C M F}
\end{array}\right\}
$$

where $\boldsymbol{\xi}_{i}^{M C M F}$, are the tracked feature positions with $i=1 . . N$ and $N$ as the number of features.

The measurement models used to update the error states are based on the developments in $[13,24,10]$. For the update by the laser altimeter measurements a modification was introduced.

In order to exploit the fact that the vehicle is landing at a well determined landing site, whose topographic elevation is known a priori, the update equations for the laser altimeter measurement are changed for the vertical descent phase of the final landing phase.

\footnotetext{
1 with the origin in the center of the Moon, z-axis pointing to the North pole, and $\mathrm{x}$ - and y-axes spanning the equatorial plane.
} 


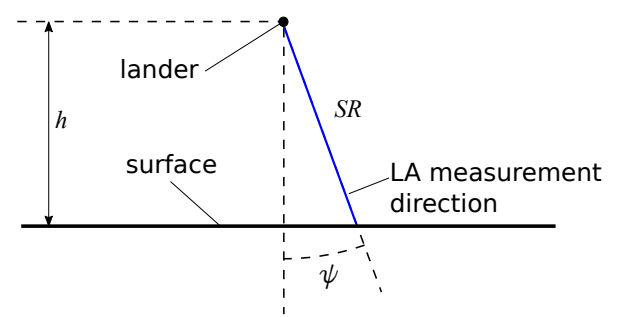

Fig. 2 Geometry for laser altimeter measurement

For simplification it is assumed that the spacecraft is landing in a horizontal plane with known altitude. In this case the height $(h)$ of the spacecraft above ground can be approximated as the difference between magnitudes of spacecraft position vector $\left(\left\|\mathbf{r}_{L A}\right\|\right)$ and landing site position vector $\left(\left\|\mathbf{r}_{L S}\right\|\right)$.

With reference to figure 2 , if the altitude of the spacecraft and the angle $\psi$ between the laser altimeter measurement direction and the position vector of the laser altimeter in MCMF frame are small enough, then the following approximate expression for the slant-range $S R$ can be used

$$
\begin{aligned}
S R & \cong h \frac{1}{\cos \psi} \\
& \cong\left(\left\|\mathbf{r}_{L A}\right\|-\left\|\mathbf{r}_{L S}\right\|\right) \frac{1}{\cos \psi}
\end{aligned}
$$

where

$$
\mathbf{r}_{L A}=\mathbf{r}+\mathbf{R}_{B}^{M C M F} \ell_{L A}^{B}
$$

is the position of the laser altimeter in MCMF frame with $\ell_{L A}^{B}$ as the lever arm of the laser altimeter with respect to the IMU, $\mathbf{r}_{L S}$ as the position of the landing site (LS) in MCMF frame and $\mathbf{r}$ as spacecraft position in MCMF frame. This could be rearranged to

$$
\cos \psi=-\boldsymbol{\zeta}^{B^{T}}\left(\mathbf{R}_{M C M F}^{B} \frac{\mathbf{r}_{L A}}{\left\|\mathbf{r}_{L A}\right\|}\right)
$$

where $\boldsymbol{\zeta}^{B}$ is the LA measurement direction unit vector in body frame. For simplification of the Jacobian the following approximation is used

$$
\frac{1}{\cos \psi}= \pm \sqrt{1+\tan ^{2} \psi} \approx \pm \sqrt{1+\sin ^{2} \psi} \approx \pm \sqrt{2-\cos ^{2} \psi}
$$

Equation (2) can be now rewritten as

$$
S R \cong \sqrt{2-\left(\frac{\mathbf{r}_{L A}^{T}}{\left\|\mathbf{r}_{L A}\right\|} \mathbf{R}_{B}^{M C M F} \zeta^{B}\right)^{2}}\left(\left\|\mathbf{r}_{L A}\right\|-\left\|\mathbf{r}_{L S}\right\|\right) .
$$

The dependency on the attitude error angles is in the rotation matrix, expressed as 


$$
\mathbf{R}_{B}^{M C M F}=\hat{\mathbf{R}}_{B}^{M C M F}\left(\mathbf{I}-\left[\boldsymbol{\theta}^{B} \times\right]\right)
$$

where $\hat{\mathbf{R}}_{B}^{M C M F}$ is the rotation matrix estimated by the filter, and $\mathbf{I}-\left[\boldsymbol{\theta}^{B} \times\right]$ is the linearized small rotation matrix.

Introducing the following terms for the sake of having more compact expressions

$$
\begin{gathered}
A=\sqrt{2-\left(\frac{\mathbf{r}_{L A}^{T}}{\left\|\mathbf{r}_{L A}\right\|} \mathbf{R}_{B}^{M C M F} \boldsymbol{\zeta}^{B}\right)^{2}} \\
B=\left\|\mathbf{r}_{L A}\right\|-\left\|\mathbf{r}_{L S}\right\| \\
C=\mathbf{R}_{B}^{M C M F} \boldsymbol{\zeta}^{B} \\
D=\frac{\mathbf{r}_{L A}^{T}}{\left\|\mathbf{r}_{L A}\right\|} \mathbf{R}_{B}^{M C M F} \boldsymbol{\zeta}^{B}
\end{gathered}
$$

the partial derivatives of equation (7) with respect to the state variables are

$$
\begin{gathered}
\frac{\partial S R}{\partial \mathbf{r}}=A \frac{\mathbf{r}_{L A}^{T}}{\left\|\mathbf{r}_{L A}\right\|}-\frac{B C^{T} D}{A}\left(\frac{1}{\left\|\mathbf{r}_{L A}\right\|} \mathbf{I}-\frac{1}{\left\|\mathbf{r}_{L A}\right\|^{3}} \mathbf{r}_{L A} \mathbf{r}_{L A}{ }^{T}\right) \\
\frac{\partial S R}{\partial \boldsymbol{\theta}^{B}}=-\frac{B D}{A} \frac{\mathbf{r}_{L A}}{\left\|\mathbf{r}_{L A}\right\|} \hat{\mathbf{R}}_{B}^{M C M F}\left[\boldsymbol{\zeta}^{B} \times\right]
\end{gathered}
$$

where it has been exploited the relation $\mathbf{a} \times \mathbf{b}=-\mathbf{b} \times \mathbf{a}$. It has to be noticed that in the derivation of equation (11) the dependency of the attitude on $\mathbf{r}_{L A}$ has been neglected, because it is much smaller with respect to the depencency on the cosine of $\psi$.

The Jacobian $\mathbf{H}_{S R}$ is:

$$
\mathbf{H}_{S R}=\left[\begin{array}{llll}
\frac{\partial S R}{\partial \mathbf{r}} & \emptyset_{1 \times 3} & \frac{\partial S R}{\partial \boldsymbol{\theta}^{B}} & \cdots
\end{array}\right]
$$

\subsection{Extension of Navigation System with Ground-based Measurements}

\subsubsection{Architecture with RF Measurements}

In order to improve the navigation accuracy, measurements of received radio signals from ground-based infrastructure (beacons, BC) are added as it is displayed in figure 3 . 


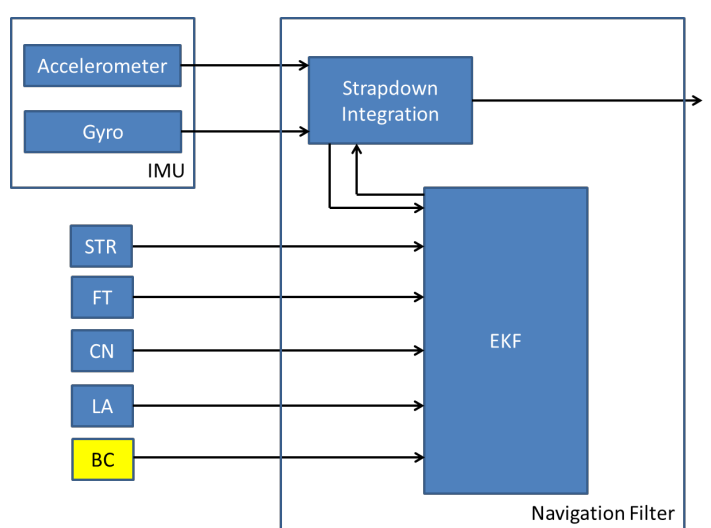

Fig. 3 Functional block diagram of SINPLEX navigation system including RF measurements of beacon (BC) signals.

RF Navigation is extensively used on Earth especially in aviation. There are many kinds of electromagnetic signals, modulation techniques and measurement methods that could be used and which are specific for the different existing navigation services. However, the information that RF navigation provides to the user can be divided into the following three main categories:

- range measurement,

- range rate measurement,

- bearing measurement.

There have been studies about the evaluation or design of possible navigation architectures for the lunar environment. Many of these studies are about the necessity of locating a user on the surface of the Moon during an extravehicular-activity (EVA), which could be the case if a lunar base is established (see e.g. $[3,5,2]$ ).

In recent years NASA has proposed for its lunar base architecture design a navigation architecture based on lunar relay satellites (LRS) and lunar communication terminals (LCT). The combination of both would provide oneand two-way range and Doppler measurement to the lander. The LCTs alone would instead provide one-way S-band Doppler and range. It is also proposed to use atomic clocks to drive the RF measurements [23] to achieve a better performance.

In $[6,7]$ the performance of a lander navigation aided by RF measurements (beacon-relative) has been studied, where the lander transponds signals from available RF assets, i.e. LRS or LCT, receiving range and range rate information from them. These measurements are inserted in a EKF together with other navigation measurements coming from IMU, star camera, velocimeter and altimeter, which is the basic sensor suite of ALHAT [8]. The study in [6, 7] has evaluated the performance of the navigation accuracy through linear covariance analyses, when on-board system is supported by a single surface beacon. The result shows that surface beacons provide the best RF measure if 
they are not located directly under the path of the lander as it approaches the landing site. A well placed beacon could support with accuracy below $10 \mathrm{~m}$ at $3-\sigma$ the final phases of landing. With the aim of improving landing accuracy in final phases, the best placement was found to be at about $2 \mathrm{~km}$ both uprange with respect to the landing site and off-track with respect to the lander groundtrack.

As far as an infrastructure will be present on the Moon the natural consequence is to make use of a beacon placed there to get measurements that would possibly increase the accuracy level of current on-board navigation architecture or decrease their weight, if some on-board sensors could be discarded while still meeting the performance requirements.

\subsubsection{Range}

The range is the measurement of the distance between the beacon and the antenna on-board of the spacecraft (see figure 4).

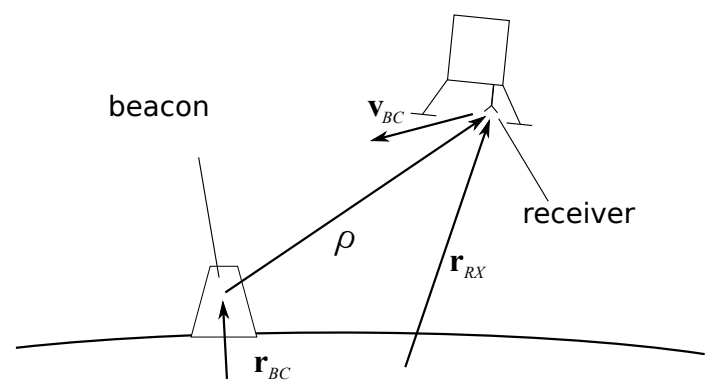

Fig. 4 Representation of range measurement between spacecraft and beacon on lunar surface

The lever arm of the receiver is first added to the position of the IMU (defining the navigation body frame) in the MCMF frame to get the position vector of the receiver (RX) in MCMF frame as

$$
\mathbf{r}_{R X}=\mathbf{r}+\mathbf{R}_{B}^{M C M F} \ell_{R X}^{B} .
$$

The vector between the beacon $(\mathrm{BC})$ and the receiver is

$$
\boldsymbol{\rho}=\mathbf{r}_{R X}-\mathbf{r}_{B C}=\mathbf{r}+\mathbf{R}_{B}^{M C M F} \ell_{R X}^{B}-\mathbf{r}_{B C} .
$$

In this study it is assumed that the clock bias is accounted as a random error. Therefore the pseudorange and range are equal. Then the range is expressed as

$$
\rho=\rho\left(\mathbf{r}, \boldsymbol{\theta}^{B}\right)=\|\boldsymbol{\rho}\|+w_{\rho}
$$

where $w_{\rho}$ is the range measurement noise. 
The partial derivatives of equation (15) with respect to the state variables are

$$
\begin{gathered}
\frac{\partial \rho}{\partial \mathbf{r}}=\frac{\partial \rho}{\partial \boldsymbol{\rho}} \frac{\partial \boldsymbol{\rho}}{\partial \mathbf{r}}=\frac{\boldsymbol{\rho}^{T}}{\rho} \\
\frac{\partial \rho}{\partial \boldsymbol{\theta}^{B}}=\frac{\partial \rho}{\partial \boldsymbol{\rho}} \frac{\partial \boldsymbol{\rho}}{\partial \boldsymbol{\theta}^{B}}=\frac{\boldsymbol{\rho}^{T}}{\rho} \mathbf{R}_{B}^{M C M F}\left[\boldsymbol{\ell}_{R X}^{B} \times\right]
\end{gathered}
$$

with

$$
\frac{\partial \boldsymbol{\rho}}{\partial \boldsymbol{\theta}^{B}}=\mathbf{R}_{B}^{M C M F}\left[\boldsymbol{\ell}_{R X}^{B} \times\right]
$$

These derivatives are properly fed into the Jacobian $\mathbf{H}_{\rho}$

$$
\mathbf{H}_{\rho}=\left[\begin{array}{llll}
\frac{\partial \rho}{\partial \mathbf{r}} & \emptyset_{1 \times 3} & \frac{\partial \rho}{\partial \boldsymbol{\theta}^{B}} & \cdots
\end{array}\right]
$$

\subsubsection{Range-Rate}

The range-rate is the relative velocity between the on-board receiver and the beacon. The following observation equation is used

$$
\dot{\rho}=\dot{\rho}\left(\mathbf{r}, \mathbf{v}, \boldsymbol{\theta}^{B}, \mathbf{b}_{g}^{B}, \mathbf{s}_{g}^{B}\right)=-\mathbf{v}_{R X}^{T} \frac{\boldsymbol{\rho}}{\rho}+w_{\dot{\rho}}
$$

where it can be shown that the velocity of the receiver in MCMF frame is

$$
\mathbf{v}_{R X}=\mathbf{v}+\left(\mathbf{R}_{B}^{M C M F}\left[\boldsymbol{\omega}_{I, B}^{B} \times\right]-\left[\boldsymbol{\omega}_{I, M}^{M} \times\right] \mathbf{R}_{B}^{M C M F}\right) \boldsymbol{\ell}_{R X}^{B} .
$$

For evaluating the angular velocity of the spacecraft the following approximation is used

$$
\boldsymbol{\omega}_{I, B}^{B} \cong \frac{\Delta \Theta^{B}}{\Delta \tau}
$$

where the angular variation is

$$
\Delta \boldsymbol{\Theta}^{B} \cong \operatorname{diag}\left(\mathbf{s}_{g}^{B}\right) \Delta \tilde{\boldsymbol{\Theta}}^{B}+\Delta \tau \mathbf{b}_{g}^{B}+w_{\Delta \boldsymbol{\Theta}} .
$$

In (23) $\Delta \tilde{\Theta}^{B}$ and $\Delta \tau$ are respectively the angular variation measured by the IMU and its sampling interval.

The following equations define the partial derivatives of the observation equation (20) with respect to the states variables.

$$
\begin{gathered}
\frac{\partial \dot{\rho}}{\partial \mathbf{r}}=\frac{\partial \dot{\rho}}{\partial \boldsymbol{\rho}} \frac{\partial \boldsymbol{\rho}}{\partial \mathbf{r}}=-\frac{\mathbf{v}_{R X}^{T}}{\rho}\left(\mathbf{I}-\frac{1}{\rho^{2}} \boldsymbol{\rho} \boldsymbol{\rho}^{T}\right) \\
\frac{\partial \dot{\rho}}{\partial \mathbf{v}}=\frac{\partial \dot{\rho}}{\partial \mathbf{v}_{R X}} \frac{\partial \mathbf{v}_{R X}}{\partial \mathbf{v}}=-\frac{\partial \rho}{\partial \mathbf{r}}
\end{gathered}
$$




$$
\begin{gathered}
\frac{\partial \dot{\rho}}{\partial \boldsymbol{\theta}^{B}}=\frac{\partial \dot{\rho}}{\partial \mathbf{v}_{R X}} \frac{\partial \mathbf{v}_{R X}}{\partial \boldsymbol{\theta}^{B}}+\frac{\partial \dot{\rho}}{\partial \boldsymbol{\rho}} \frac{\partial \boldsymbol{\rho}}{\partial \boldsymbol{\theta}^{B}}= \\
-\frac{\partial \rho}{\partial \mathbf{r}}\left(\mathbf{R}_{B}^{M C M F}\left[\left(\boldsymbol{\omega}_{I, B}^{B} \times \boldsymbol{\ell}_{R X}^{B}\right) \times\right]-\left[\boldsymbol{\omega}_{I, M}^{M} \times\right] \mathbf{R}_{B}^{M C M F}\left[\boldsymbol{\ell}_{R X}^{B} \times\right]\right)+\frac{\partial \dot{\rho}}{\partial \boldsymbol{\rho}} \frac{\partial \boldsymbol{\rho}}{\partial \boldsymbol{\theta}^{B}} \\
\frac{\partial \dot{\rho}}{\partial \mathbf{b}_{g}^{B}}=\frac{\partial \dot{\rho}}{\partial \mathbf{v}_{R X}} \frac{\partial \mathbf{v}_{R X}}{\partial \mathbf{b}_{g}^{B}}=\frac{\partial \rho}{\partial \mathbf{r}} \mathbf{R}_{B}^{M C M F}\left[\ell_{R X}^{B} \times\right] \\
\frac{\partial \dot{\rho}}{\partial \mathbf{s}_{g}^{B}}=\frac{\partial \dot{\rho}}{\partial \mathbf{v}_{R X}} \frac{\partial \mathbf{v}_{R X}}{\partial \mathbf{s}_{g}^{B}}=\frac{\partial \dot{\rho}}{\partial \mathbf{b}_{g}^{B}} \operatorname{diag}\left(\Delta \tilde{\boldsymbol{\Theta}}^{B}\right) \frac{1}{\Delta \tau}
\end{gathered}
$$

The Jacobian $\mathbf{H}_{\dot{\rho}}$ for the filter update is

$$
\mathbf{H}_{\dot{\rho}}=\left[\begin{array}{lllllll}
\frac{\partial \dot{\rho}}{\partial \mathbf{r}} & \frac{\partial \dot{\rho}}{\partial \mathbf{v}} & \frac{\partial \dot{\rho}}{\partial \boldsymbol{\theta}^{B}} & \emptyset_{1 \times 6} & \frac{\partial \dot{\rho}}{\partial \mathbf{b}_{g}^{B}} & \frac{\partial \dot{\rho}}{\partial \mathbf{s}_{g}^{B}} & \cdots
\end{array}\right] .
$$

\subsubsection{Bearing}

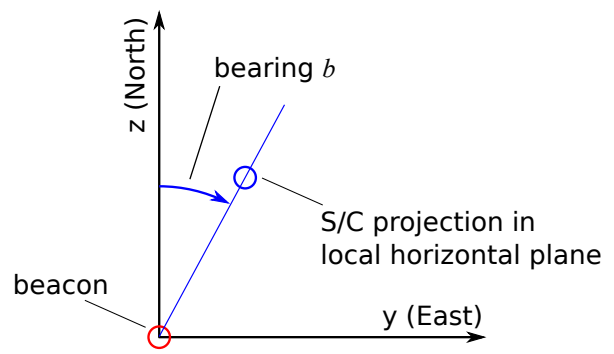

Fig. 5 Definition of bearing measurement

The bearing is here defined as the Azimuth which is the angle between North direction and the projection of the vector between spacecraft position and beacon position on the local horizontal (LH) plane (figure 5). It is meant as a similar measurement to what VHF Omni Directional Radio Range (VOR) systems perform on Earth [16]. In order to compute the bearing, the position of the receiver has to be rotated in the local horizontal frame of the beacon with respect to whom the bearing is measured.

$$
\mathbf{r}_{R X}^{L H}=\mathbf{R}_{M C M F}^{L H} \mathbf{r}_{R X}
$$

In order to project the position vector in the E-N plane, the following matrix is introduced

$$
\mathbf{L}=\left[\begin{array}{lll}
0 & 0 & 0 \\
0 & 1 & 0 \\
0 & 0 & 1
\end{array}\right]
$$


Having introduced this matrix, the bearing can be computed as the arccosine of

$$
X=\mathbf{n}^{T} \frac{\mathbf{L} \mathbf{r}_{R X}^{L H}}{\left\|\mathbf{L} \mathbf{r}_{R X}^{L H}\right\|}
$$

where $\mathbf{n}$ is the unit vector parallel to E-N plane and heading to the north.

Being the arccosine function defined in the interval $[0, \pi]$, the following relations are introduced in order to have the bearing observation equation defined in the interval $[0,2 \pi]$

$$
\begin{gathered}
y_{R X}^{L H} \geq 0 \Longrightarrow b=b\left(\mathbf{r}, \boldsymbol{\theta}^{B}\right)=\arccos (X)+w_{b} \\
y_{R X}^{L H}<0 \Longrightarrow b=b\left(\mathbf{r}, \boldsymbol{\theta}^{B}\right)=2 \pi-\arccos (X)+w_{b} .
\end{gathered}
$$

The partial derivatives needed by the eEKF are

$$
\begin{gathered}
\frac{\partial b}{\partial \mathbf{r}}=\frac{\partial b}{\partial X} \frac{\partial X}{\partial \mathbf{r}_{R X}^{L H}} \frac{\partial \mathbf{r}_{R X}^{L H}}{\partial \mathbf{r}}= \\
\mp \frac{1}{\sqrt{1-X^{2}}} \mathbf{n}^{T}\left(\frac{1}{\left\|\mathbf{L} \mathbf{r}_{R X}^{L H}\right\|} \mathbf{I}-\frac{\left(\mathbf{L} \mathbf{r}_{R X}^{L H}\right)\left(\mathbf{L} \mathbf{r}_{R X}^{L H}\right)^{T}}{\left\|\mathbf{L} \mathbf{r}_{R X}^{L H}\right\|^{3}}\right) \mathbf{R}_{M C M F}^{L H} \\
\frac{\partial b}{\partial \boldsymbol{\theta}^{B}}=\frac{\partial b}{\partial X} \frac{\partial X}{\partial \mathbf{r}_{R X}^{L H}} \frac{\partial \mathbf{r}_{R X}^{L H}}{\partial \boldsymbol{\theta}^{B}}=\frac{\partial b}{\partial \mathbf{r}} \mathbf{R}_{B}^{M C M F}\left[\ell_{R X}^{B} \times\right] .
\end{gathered}
$$

In equation (35) the negative or positive sign is respectively related to the application of equations (33) or (34). The $\mathbf{H}$ matrix becomes

$$
\mathbf{H}_{b}=\left[\begin{array}{llll}
\frac{\partial b}{\partial \mathbf{r}} & \emptyset_{1 \times 3} & \frac{\partial b}{\partial \boldsymbol{\theta}^{B}} & \cdots
\end{array}\right] .
$$

\subsubsection{Visibility Model}

The measurements from a beacon are only available when the lander is inside the visibility window.

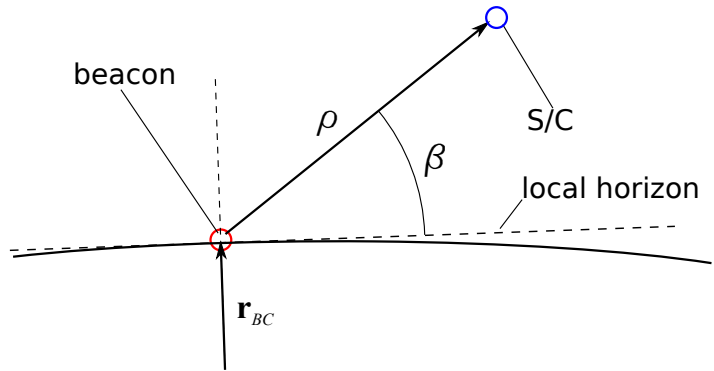

Fig. 6 Visibility model 
With reference to figure 6 , the condition for the visibility is

$$
\frac{\pi}{2}-\arccos \left(\frac{\boldsymbol{\rho}^{T} \mathbf{r}_{B C}}{\|\boldsymbol{\rho}\|\left\|\mathbf{r}_{B C}\right\|}\right)=\beta \geq \beta_{L I M} .
$$

Since the real morphology of the surface is unknown, it has been assumed that a minimum elevation angle $\left(\beta_{L I M}\right)$ of 10 degrees with respect to the horizon is needed in order to consider the lander visible from the beacon and the update valid.

\section{Scenario and Analyses Method}

\subsection{Definition of Lunar Landing Scenario}

The scenario is a lunar soft landing. The landing trajectory is adapted from the project Autonomous Terrain based Optical Navigation (ATON) of DLR. The trajectory starts with a maneuver to be inserted in a 100x10 km descent orbit from a 100x100 km polar orbit.At perigee the powered descent is initiated and at $2 \mathrm{~km}$ altitude at high gate when the landing site is in view the landing phase starts. Finally a vertical descent is performed from around $1 \mathrm{~km}$ altitude (figure $7(\mathrm{a})$ )

The trajectories have been computed under the assumption that the throttling of the thrusters is limited and that the main thrust modulation has to be provided by switching off one or pairs selected from the five engines. The results and methods are described in $[21,17]$.

More in detail, the descent orbit is chosen such to provide optimal lighting condition for optical navigation. A variable thrust is used to slow down the lander as it descends towards the landing site which is located near the equator. A small acceleration is also given during the final phase of powered descent in cross-range direction in order to cope with the lunar surface rotation. Figure 8 (a) shows the specific force profile.

Throughout the powered descent the attitude is constrained by the thrust profile. It is assumed to have the antenna used for the beacon navigation near the navigation camera on the bottom surface of the lander that it is directly visible from the beacons during powered descent. Figure 8(b) presents the angular velocity profile.

The simulation starts with the descent orbit burn and ends at an altitude of $1 \mathrm{~m}$ above the landings site. So it includes all parts of the landing. The final velocity is less than $0.5 \mathrm{~m} / \mathrm{s}$. For later analyses it is useful to show the powered descent groundtrack on the local horizontal plane, in order to better understand some considerations during the analyses (figure $7(\mathrm{~b})$ ).

\subsection{Simulation Setup}

The results that will be later presented are the outcome of Monte Carlo analyses. The number of runs has been chosen to be 100. In each of the 100 runs 

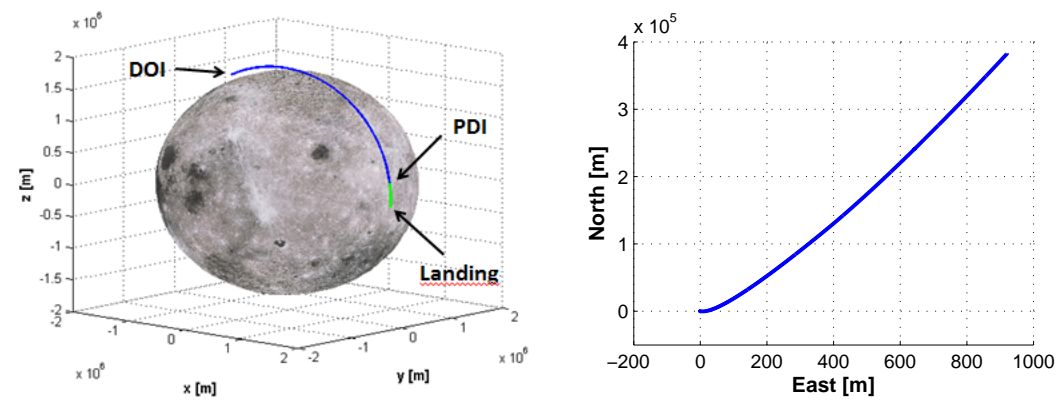

Fig. 7 Landing trajectory (left) and groundtrack of final phase in local horizontal plane (right)
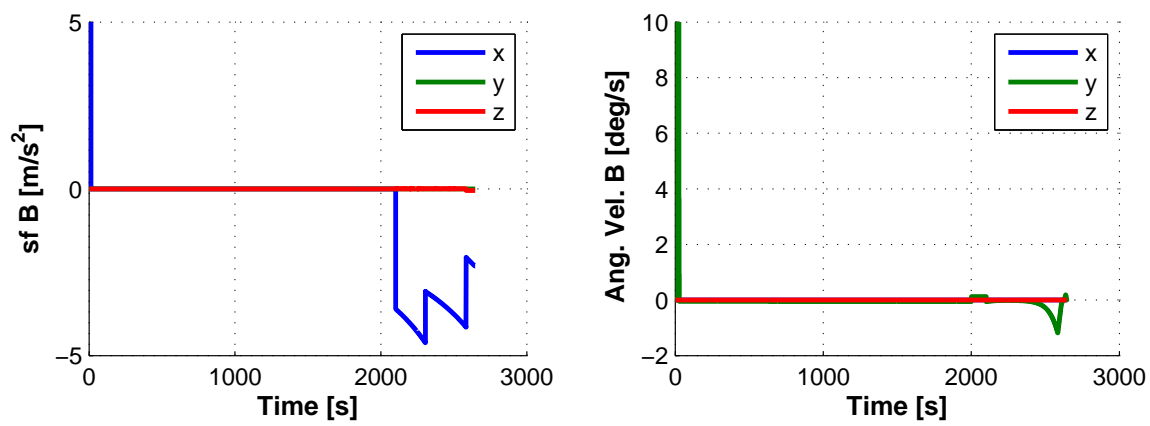

Fig. 8 Control profile

the random seeds for all noise generators as well as the systematic errors of the sensors (e.g. bias, scale factor) have been randomly changed. First of all a pool of 100 simulation runs without the RF measurement updates has been generated in order to have reference navigation solutions for further analyses. These 100 different navigation solutions without RF measurements are used as the initial navigation state for the Kalman filters which are using the RF measurements. Thus an initial navigation error dispersion is achieved for the subsequent Monte Carlo simulations for the different beacon locations.

First the results of the integration of the true dynamics is used to generate the sensor measurements for each simulation run. This part of the simulation is performed in a dedicated Simulink model which contains the truth model and the sensor models. Then these results are processed by the navigation algorithm which is implemented in a Matlab script.

After the Monte Carlo simulation 100 navigation solutions are available. From this set a worst case navigation solution is extracted. In fact, it has been considered appropriate to perform trade-offs analyses on worst case navigation solutions, comparing them to $3-\sigma$ requirements (see section 3.4.1). This worst case is evaluated extracting the maximum absolute navigation error per each 
time instant $i$ of the navigation solution for all runs $N_{M C}$. The worst case error $\Delta E_{i}$ can be written as

$$
\Delta E_{i}=\max \left(\left|\Delta e_{i}\right|\right)_{j=1}^{N_{M C}}
$$

where $\Delta e_{i}$ is the error between the result of the navigation solution at a given time instant $i$ and the truth. The variance of the 100 runs has also been evaluated and compared with the covariances, in order to check if the covariances set in the EKF were appropriately tuned.

For the analyses involving the beacons the same process has been applied. The output of each Monte Carlo analysis for a given beacon configuration under study, was then processed through equation (39), in the same way the baseline worst case solution without beacon has been obtained.

\subsection{Error Models}

In this section the error models for the measurements in the navigation system will be defined.

\subsubsection{Baseline Sensor Suite}

Table 1 lists the parameters for the Inertial Measurement Unit (IMU) used in the simulations. In table 2 the noise figures associated to the rest of the baseline sensor suite are shown. Studies are on-going to characterize the noise associated to crater navigation. For this work the noise related to the position determination of the crater navigation $(\mathrm{CN})$ has been set to be normally distributed with standard deviation proportional to the slant-range. The crater navigation is assumed to stop working below an altitude of $10 \mathrm{~km}$. The reason is that currently available digital elevation maps (DEM) do not allow to generate accurate crater catalogues usable below $10 \mathrm{~km}$ of altitude because of missing resolution and accuracy. This is a conservative assumption since it is likely to have higher resolution maps in the near future.

Both the error in the crater navigation and feature tracker (FT) algorithm were tuned from comparison of the obtained navigation solution with the results from the ATON project where simulated images were processed [1].

\subsubsection{Beacon}

In table 3 the noise figures associated to the measurements from the beacons are shown. When selecting these values a conservative approach has been considered.

For range measurements the chosen level of the range noise is comparable with the user equivalent range error (UERE) for the GPS C/A code with no selective availability. The dominant error in UERE is related to the atmosphere and ionosphere (see [22]). Since the lunar atmosphere is negligible it could be expected then that the level of noise experienced in the scenario is much less. 
Table 1 IMU parameters $(1-\sigma)$

\begin{tabular}{lcc}
\hline Parameter & Value & Units \\
\hline Accelerometer & & \\
- Bias level & 25.5 & $\mathrm{mg}$ \\
- Bias stability & 1.5 & $\mathrm{mg}$ \\
- Random walk & 0.0106 & $\mathrm{~m} / \mathrm{s} / \sqrt{h r}$ \\
- Scale factor error & $3.33 \cdot 10^{-4}$ & - \\
- Scale factor error stability & $1.67 \cdot 10^{-6}$ & - \\
\hline Gyroscope & & \\
- Bias level & 825 & $\mathrm{deg} / \mathrm{hr}$ \\
- Bias stability & 4 & $\mathrm{deg} / \mathrm{hr}$ \\
- Random walk & 0.9 & $\mathrm{deg} / \sqrt{h r}$ \\
- Scale factor error & $3.33 \cdot 10^{-5}$ & - \\
- Scale factor error stability & $10^{-6}$ & - \\
\hline
\end{tabular}

Table 2 Error parameters (1- $\sigma$ ) for star tracker (STR), crater navigation $(\mathrm{CN})$, feature tracker (FT) and laser altimeter (LA).

\begin{tabular}{lcc}
\hline Parameter & Value & Units \\
\hline STR accuracy & 9.1 & arcsec \\
CN accuracy & $3 \%$ of slant-range & m \\
FT accuracy & 1 & pixel \\
LA accuracy & 0.04 & $\mathrm{~m}$ \\
\hline
\end{tabular}

Table 3 Beacon measurements error parameters (1- $\sigma)$

\begin{tabular}{lcc}
\hline Parameter & Value & Units \\
\hline Range & 10 & $\mathrm{~m}$ \\
Range-rate & 0.1 & $\mathrm{~m} / \mathrm{s}$ \\
Bearing & 1.4 & $\mathrm{deg}$ \\
\hline
\end{tabular}

Moreover, studies about relative navigation system for space applications using GPS receivers have shown errors in the relative state vector components of typically $0.5 \mathrm{~m}$ and $1 \mathrm{~cm} / \mathrm{s}$ in hardware-in-the-loop simulations [19]. Eventually, with the aim of not underestimating the errors and considering all the uncertainties in the observation models used, the noise figures selected for range and range-rate can be considered a good compromise.

Considering the bearing, the literature concerning VOR navigation system claims that it is not a precision aid. A 2- $\sigma$ predictable accuracy of $1.4 \mathrm{deg}$ is reported although it seems that seldom worst case errors of around $\pm 4-5 \mathrm{deg}$ are possible [9]. In this study, for similar considerations regarding the choice of range and range-rate noise, it has been decided to use the value reported in table 3 for the bearing measurement noise. 
A further reference on relative range and bearing accuracies can be taken from the flight results of the Formation Flying Radio Frequency (FFRF) instrument which was tested on the PRISMA mission. It uses RF signals between up to 4 satellites to acquire relative position, velocity and line of sight over a range of up to $30 \mathrm{~km}$. A modified version of the system could be a potential technical implementation of a beacon system. In [12] the flight test results from the PRISMA mission with accuracies in the order of centimeters and less than 1 degree are reported. Both values confirm that the numbers chosen in table 3 are conservative.

One aspect not yet considered in this study is the impact of the uncertain knowledge about the beacon position. In [15] the positioning of the Chang'E-3 lunar lander is achieved with errors in the order of a few meters. It is assumed that the same accuracy can be achieved for a beacon on the lunar surface. Since this achievable positioning accuracy is a fraction of the assumed ranging error, in a first approach the beacon positioning uncertainty is assumed to be included in the errors in table 3. Thus an analysis of most suitable beacon locations could be done. A detailed analysis on the impact of the beacon positioning error would be subject of a further study.

\subsection{Navigation Requirements and Evaluation Method}

\subsubsection{Navigation Accuracy Requirement Profile}

The trade-offs presented in this paper require the definition of an evaluation function useful to compare one configuration to another and to the baseline. For that purpose profiles for the required 3- $\sigma$ navigation accuracy for position and velocity have been generated which guarantee a successful landing.

To generate the profiles, requirements for time instants corresponding to relevant landing phases have been collected. The values in detail can be seen in table 4 . The values have been derived from relevant lunar landing requirements $[11,14,26,8]$. They are given in downrange, crossrange and altitude (DCA $)^{2}$. The same coordinates will be used for the analysis of results later in this paper.

Table 4 3- $\sigma$ navigation accuracy requirement from Powered Descent Initiate (PDI), through High Gate (HG) to landing

\begin{tabular}{lcccccc}
\hline & \multicolumn{2}{c}{ PDI } & \multicolumn{2}{c}{ HG } & \multicolumn{2}{c}{ Landing } \\
\hline & $\Delta \bar{E}_{r}[m]$ & $\Delta \bar{E}_{v}[m / s]$ & $\Delta \bar{E}_{r}[m]$ & $\Delta \bar{E}_{v}[m / s]$ & $\Delta \bar{E}_{r}[m]$ & $\Delta \bar{E}_{v}[m / s]$ \\
\hline $\mathrm{DR}$ & 2000 & 1 & 100 & 0.5 & 10 & 0.1 \\
$\mathrm{CR}$ & 2000 & 1 & 100 & 0.5 & 10 & 0.1 \\
$\mathrm{~A}$ & 200 & 1 & 20 & 0.5 & 0.5 & 0.1 \\
\hline
\end{tabular}

2 where the downrange direction points in nominal flight direction, altitude is aligned along the local vertical and crossrange is perpendicular to both. 
Based on the requirements in table 4 the derived profiles for the navigation accuracy requirements are shown in figure 9. This study focuses on the navigation performance in the most critical phase the powered descent. Therefore the profiles start at the Powered Descent Initiate (PDI).
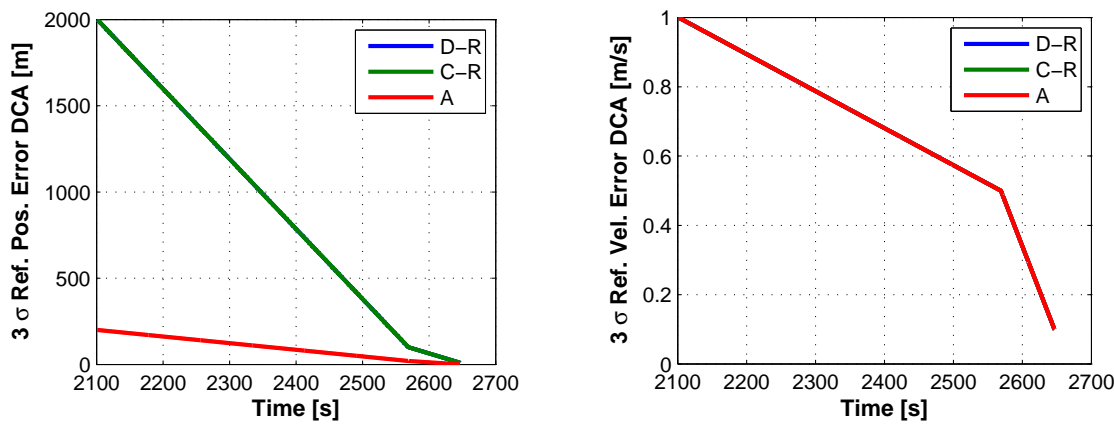

Fig. 9 Navigation accuracy requirement profiles

It can be noticed that higher accuracy is requested as the landing site is approached. The velocity profiles are the same for the down-range, cross-range and altitude, while the position error in the altitude has to be generally one order of magnitude smaller than in the other components. The $3-\sigma$ position accuracy required at landing is challenging for current technology, but in this study it was considered as mandatory if a precise landing relatively to a well defined landing site is wanted.

\subsubsection{Evaluation Functions}

The general form for the evaluation functions used in the upcoming analyses is

$$
J=\frac{1}{N} \sum_{i=I_{0}}^{I_{F}}\left(\frac{\Delta E_{i}}{\Delta \bar{E}_{i}}\right)^{2}
$$

where $\Delta \bar{E}_{i}$ is the 3 - $\sigma$ navigation accuracy requirement corresponding to the assessed navigation error; $i$ is the index for the time of the navigation solutions; $I_{0}$ and $I_{F}$ are respectively the initial and final time indexes corresponding to the time interval in which the evaluation function has to be evaluated; $N$ is the number of samples in the interval.

Equation (40) is basically similar to an integral of the worst case navigation solution errors (equation (39)) weighted with the navigation accuracy requirements $\Delta \bar{E}_{i}$ defined in the previous subsection. The term inside the summation is squared so that everything that exceeds the navigation accuracy requirements is weighted more. 
A number of four intervals have been defined, in which to evaluate the evaluation functions. The main interval goes from the first valid beacon update (called $t_{V I S}$ ) to the landing (evaluation function class $J$ ). This interval is meant to study the performance for the whole period in which the beacon measurements are impacting on the navigation solution.

The other three are sub-intervals of the main one. They are needed in order to enter deeper in the evaluation of the performance of a given configuration. This way it is possible to see how the studied option is impacting on the different relevant phases of the landing. The three subintervals are:

1. The interval corresponding to evaluation function class $J_{1}$ starts at $t_{V I S}$ and ends at $t_{M I D}$, which is defined as the mid point between $t_{V I S}$ and $t_{H G}$, i.e. the time in which $\mathrm{s} / \mathrm{c}$ reaches high gate.

2. The interval for $J_{2}$ starts at $t_{M I D}$ and ends at $t_{H G}$.

3. The interval related to $J_{3}$ goes from $t_{H G}$ to the landing.

In total 24 independent evaluation functions are available. In table 5 a notation overview is shown for the evaluation function class 1 , in order to make the reader to understand the notation used in the analysis.

Before discussing the results it should be noted that a value of the evaluation function above 1 signals indicates that the analysed configuration is performing worse than the requirements. If the evaluation function value is below 1 the requirements are met on average.

Table 5 Evaluation function notation (example for $J_{1}$ )

\begin{tabular}{ccccc}
\hline & DR & CR & A & Total \\
\hline Position & $J_{1, r}^{D R}$ & $J_{1, r}^{C R}$ & $J_{1, r}^{A}$ & $J_{1, r}^{t o t}=J_{1, r}^{D R}+J_{1, r}^{C R}+J_{1, r}^{A}$ \\
Velocity & $J_{1, v}^{D R}$ & $J_{1, v}^{C R}$ & $J_{1, v}^{A}$ & $J_{1, v}^{t o t}=J_{1, v}^{D R}+J_{1, v}^{C R}+J_{1, v}^{A}$ \\
\hline
\end{tabular}

\subsection{Constraints for Beacon Location}

In principle, it could be of interest to make the analysis for all possible locations as long as the beacon is visible for a part of the trajectory.

In figure 10 (b) it is possible to see that the longest visibility is for a beacon placed in the landing site which is somehow expected because the lander is slower in the final phase of the powered descent. It is also expected that the smaller the visibility window is, the smaller is the impact on the navigation. Following that line of thought it is hard to believe that an effort to place a beacon on the lunar surface is made, if that beacon is far from the landing site, or from the landing trajectory. However, it is clear from the previous analyses of the baseline solution (see figure 12) that the part of the trajectory requiring more improvements is the final phase of the powered descent, where the requirements for positioning are more challenging. 
For all these reasons beacons too distant from the landing site have not been taken into account. Therefore a square grid with the landing site in the middle and a base length of $20 \mathrm{~km}$ along the North and East directions has been chosen. In figure 10(a) the points of the grid are shown for which the Monte Carlo analysis will be performed. It can be seen that the points are uniformly distributed and the beacons in the grid are quite dense (121 points). There are also four curves shown which represent the $\pm 3 \sigma$ (in green) and the $\pm(2 \mathrm{~km}+3 \sigma)$ (in red) boundaries. These boundaries come from NASA's recommendations about avoiding to land nearer than $2 \mathrm{~km}$ from objects on the surface to be protected and to make sure not to intersect the landing groundtrack with $3 \sigma$ uncertainty (either up-range and down-range) within this $2 \mathrm{~km}$ avoidance circle around the object [20]. The avoided objects in this scenario are the beacons which could be harmed either by the plume of the lander, by dust and particles on the soil projected at high velocity by the plume impinging on the surface, or by a potential loss of the lander during landing, which could fall near it, even destroying it [20].

However, it can be noticed from figure 10(a), that also points inside the avoidance area have been inserted in the trade-off analysis. This is done in order to have a more uniform data distribution for the data processing and interpolations, and for the interest in checking what happens for beacons located very near to the landing site.
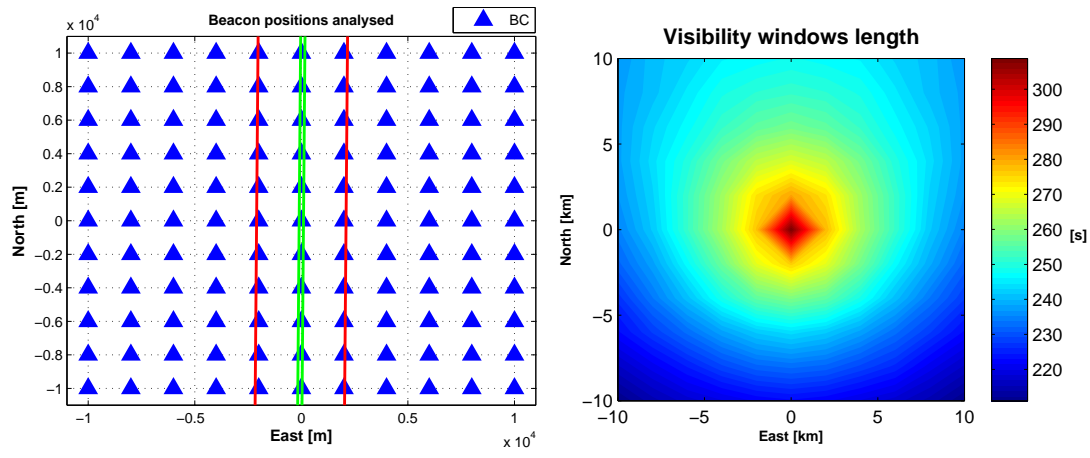

Fig. 10 Test grid for the single beacon configuration (left); Visibility windows for the tested beacons in the grid (right)

\section{Simulation Results}

\subsection{Baseline Navigation Solution}

In this section the baseline worst case navigation solution is presented (figures 11 and 12). It is the output of the Monte Carlo analysis for a navigation system 
with onboard sensors (e.g. SINPLEX, see section 2.1) and without the use of beacon updates.
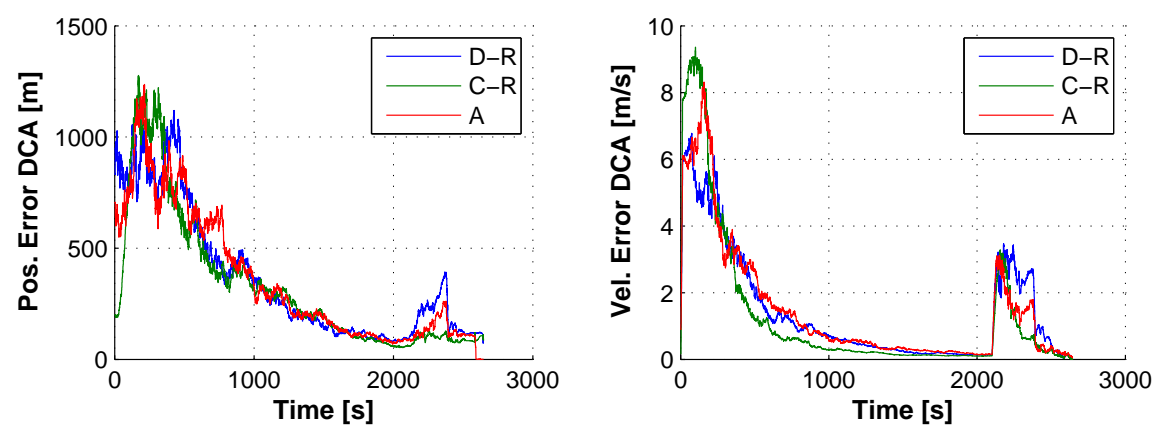

Fig. 11 Baseline worst case absolute errors (DCA): Position (left), velocity (right)

It can be seen that the navigation solution follows the high noise in the crater navigation at the beginning of the simulation (figures 11(a) and 11(b)), when the spacecraft is entering in the descent orbit because in that phase the navigation is relying only on the IMU and the crater navigation. With time the navigation solution improves since the crater navigation becomes more accurate at lower altitudes. Shortly before powered descent initiate (PDI) also laser altimeter and feature tracker measurements are updating the filter. As powered descent starts it is possible to notice an increase of the errors, especially for the velocity (figures 12(a) and 12(b)).
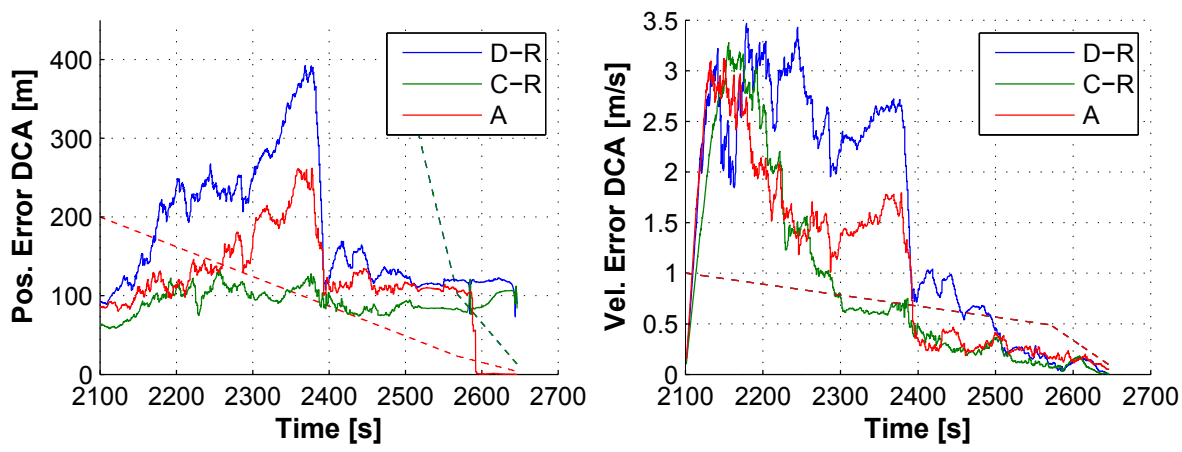

Fig. 12 Zoom of figure 11 for powered descent - baseline worst case absolute errors (DCA): Position (left), velocity (right). The dashed lines represent the requirements from table 4 and figure 9.

The error in the crossrange position and velocity stays more or less constant throughout the whole powered descent. This is the contribution of the feature tracking which is more sensitive to the crossrange. The errors in down-range 
and altitude are larger. They reach a maximum around the middle of the powered descent which corresponds approximately to the point of maximum altitude during powered descent. At the end of the powered descent below an altitude of $10 \mathrm{~km}$ when the crater navigation does not update the position anymore, the position error reaches values of around $100 \mathrm{~m}$ for crossrange and downrange position components. For the altitude the error drops when the landing site is in view and the laser altimeter measurements can be used (figure $12(\mathrm{a})$ ). The velocity error is approaching low values of around $0.1 \mathrm{~m} / \mathrm{s}$ as the landing site is reached (figure 12(b)).

Comparing figure 12(a) with figure 9 it can be seen that the position error output of the baseline navigation is for most of the time below the imposed values of the navigation accuracy requirement profile, except in the last phase for the downrange and crossrange direction, and for the altitude in the phase from the middle of the powered descent until the laser altimeter improves its determination. The final error is much larger than what is required for a precise landing relative to a given landing site.

4.2 One Beacon with Range, Range-rate and Bearing Measurements (Case A)

In this section, the trade-off analysis results on the impact of the three new measurements (range, range-rate, bearing) from a single beacon and of its location are presented. The results have been generated with 100 Monte Carlo runs for each of the 121 grid points for the potential beacon location. Thus 12,100 simulations have been executed.

In figures 13 to 15 the results of the analyses are shown. Starting with the grid of uniform distributed data, the available values have been exploited to create filled contour plots which allow to see trends. Above the color bars the corresponding values of the same scenario but without RF measurement (see section 4.1) are shown for information.

The first and most important conclusion is that wherever the beacon is positioned inside the test grid, the overall impact on the navigation solution is positive, in particular for what concerns the position determination. In fact, from figures $13(\mathrm{~d})$ to $15(\mathrm{~d})$, it is possible to see that the values of the various $J^{t o t}$ evaluation functions are always below the ones evaluated for the baseline. The best improvement is in the position determination during the last phase, starting from high gate $\left(J_{3, r}\right)$. It is possible to see from figure $15(\mathrm{~d})$ that the values drop from 32 without beacon to around 0 with beacon, for beacons near to the landing site, but in any case they are never above 4 .

The velocity does not resent of the same level of improvement which is also due to the fact that the original sensor suite is already performing well with respect to the defined navigation accuracy requirement.

Looking at figures 13 and 15 for the downrange but also for the altitude components, it is possible to see a common trend. The related evaluation functions assume larger values going down-range, i.e. South of the landing site, which is somehow correlated with the visibility interval which naturally 
decreases going downrange, as figure 10(b) confirms. The minimums are instead generally located along the landing ground track and a bit up-range. The trend assumed by these position evaluation functions related to the altitude is then reflected in the $J^{\text {tot }}$ where the absolute values are larger than in the downrange and crossrange components. This is a direct consequence of the fact that the requirements in altitude errors are one order of magnitude more accurate (see table 4 ).

It was somehow then expected to find a symmetry with respect to the North axis in the contour plots since the landing trajectory is substantially coming from North. Looking through the figures, not for all evaluation functions a symmetry can be found. It seems that especially for crossrange the fact that the lander is slightly coming from East plays a role. However, looking at the total evaluation function $J^{\text {tot }}$ for position the performance shows the expected symmetry left and right with respect to the North axis although it is not perfectly symmetric.
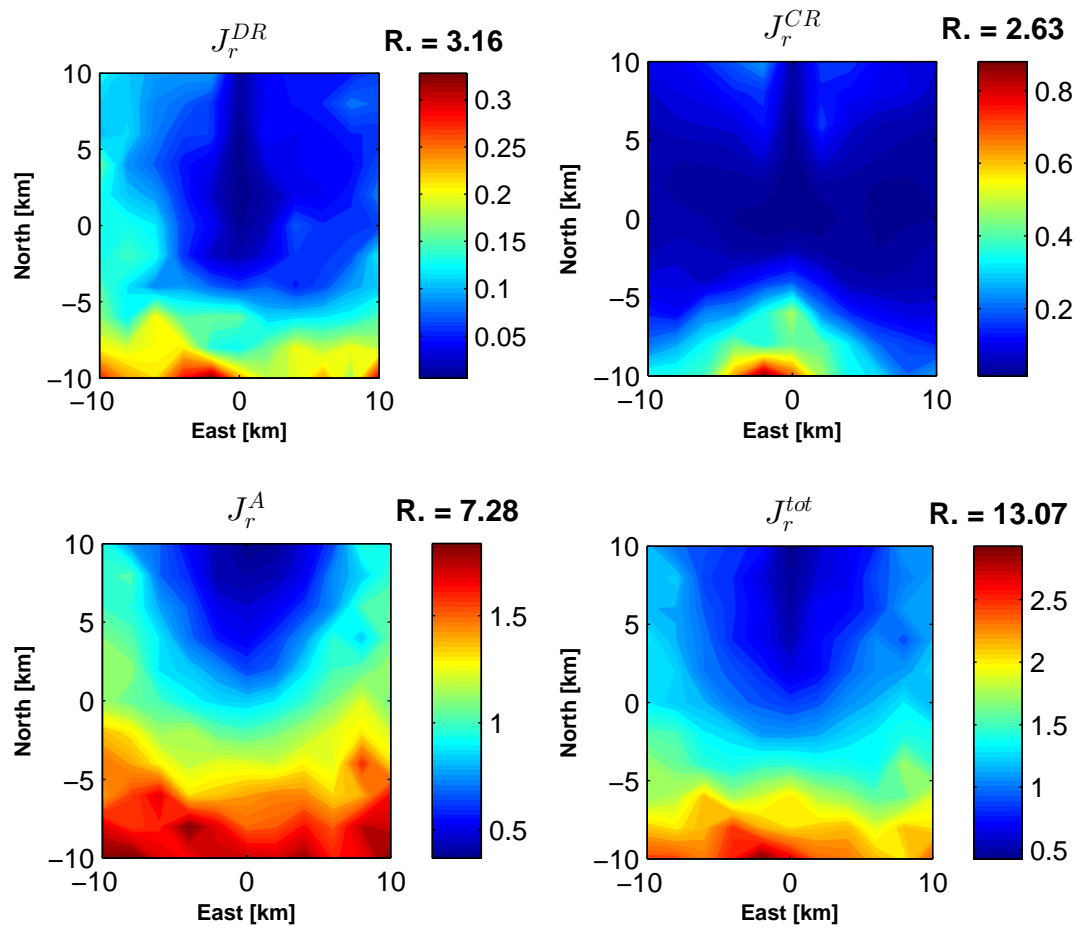

Fig. $13 J_{r}$ evaluation functions (case A). The value above each color bar shows the same cost function value for the baseline case without RF measurements.

There is a general improvement in adding the beacon updates to the baseline on-board navigation system. An overall look at figures 13 to $15(\mathrm{~d})$ suggests that the best area in which to locate the beacon is along the landing ground- 

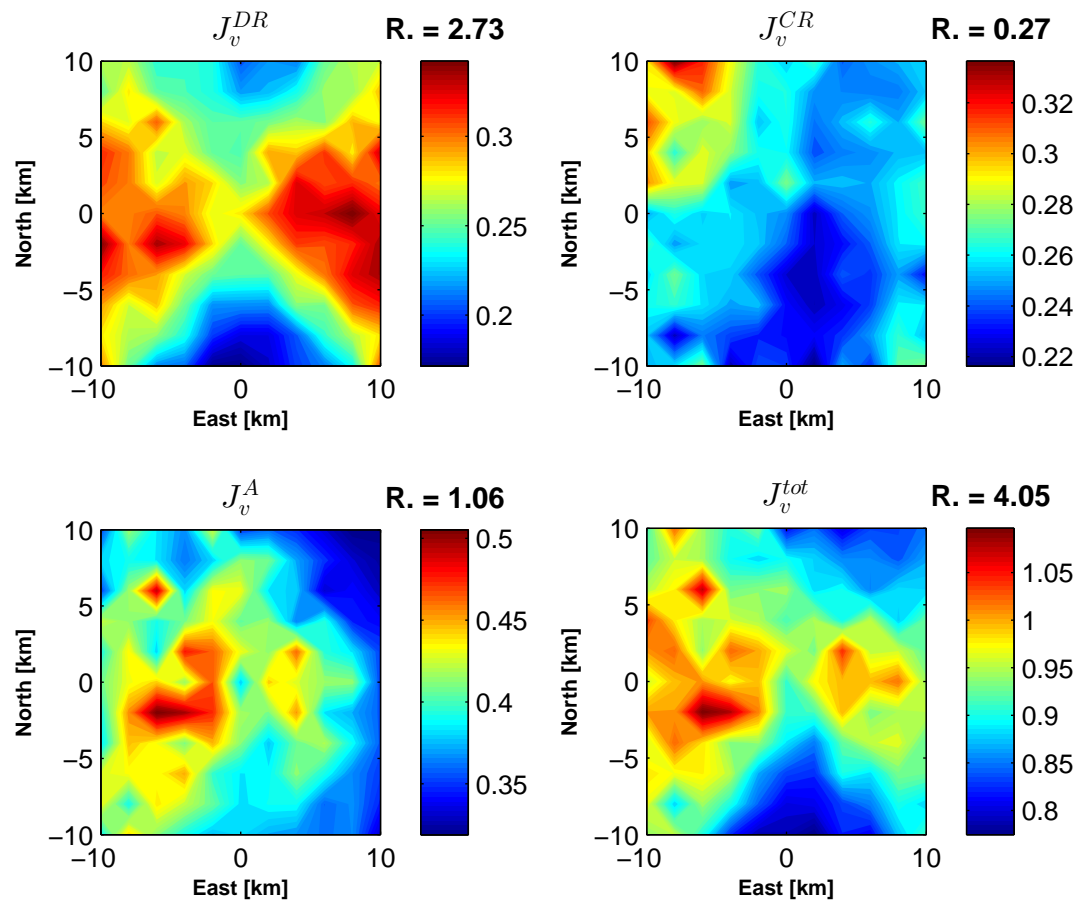

Fig. $14 J_{v}$ evaluation functions (case A). The value above each color bar shows the same cost function value for the baseline case without RF measurements.

track, slightly up-range with respect to the landing site. This area guarantees low values of evaluation functions and low values of the final position errors meeting the requirements. This result is somehow expected since:

- the best configuration for the down-range improvement is to position the beacon along the ground-track while the spacecraft is approaching (in this case the range and range-rate measured are more or less aligned with the downrange position and velocity);

- the best configuration for the altitude is having the beacon under the spacecraft, since in that case the range coincides with the altitude;

- the presence of the bearing measurement gives information from which cross-range can be retrieved, also when the beacon is placed in that zone. In fact, looking at the derivation of the observation model in equations (31) to (34), the bearing depends on East and North component $y^{L H}$ and $z^{L H}$. Therefore it allows to retrieve information both in downrange and crossrange independent from its position.

Unfortunately, the beacon position providing the best navigation performance falls inside the area that should be avoided for reasons discussed in section 3.5 .

Taking into account the final position error in downrange and crossrange, figures 16(a) and 16(b) prove that these worst case errors are below the re- 

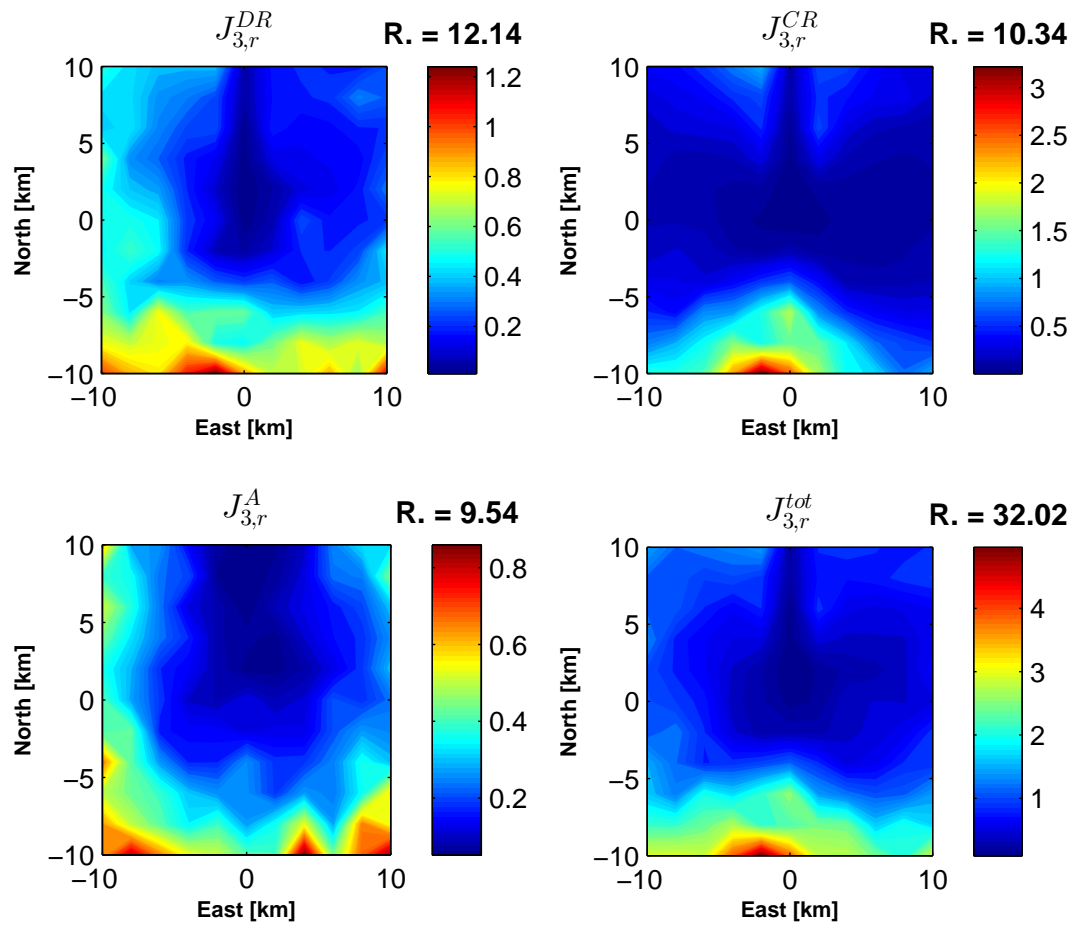

Fig. $15 J_{3, r}$ evaluation functions (case A). The value above each color bar shows the same cost function value for the baseline case without RF measurements.
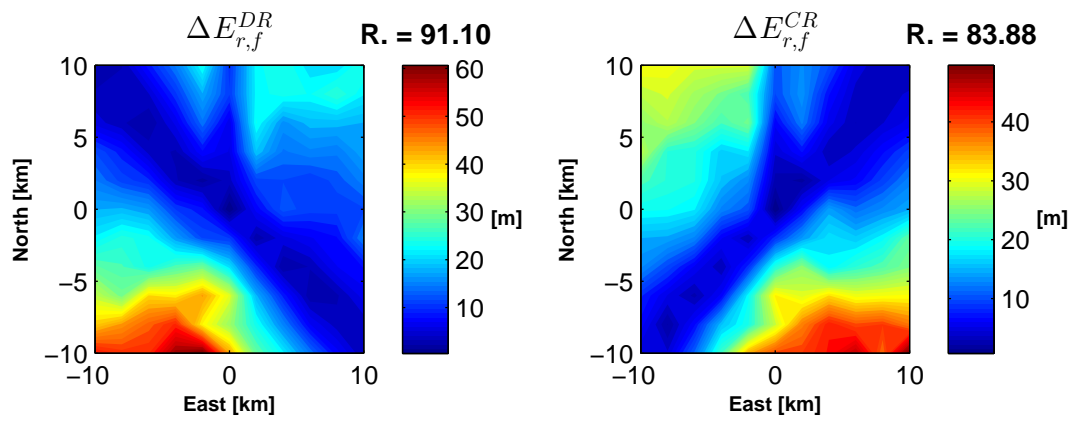

Fig. 16 Final position errors $\Delta E_{r, f}$ (case A). The value above each color bar shows the final position error for the baseline case without RF measurements.

quired $10 \mathrm{~m}$ at 3- $\sigma$ not everywhere in the grid, and in some areas located down and cross-range they reach values around $60 \mathrm{~m}$, i.e. much higher.

In particular, it seems that these errors increase with the distance of the beacon to the landing site which is expected for the following reasons:

- for those beacons the visibility window does not cover the very last part of the navigation, when the spacecraft falls below the horizon, and 
- the bearing is a more precise update for the position and velocity estimation when the spacecraft is nearer to the beacon.

These errors are also small almost everywhere along the bisectors North-West to South-East for the downrange and North-East to South-West for the crossrange. This behavior, symmetric due to the orthogonality of the two components, is connected to the direction from which the landing site is approached. The landing site is in fact approached from South-South-East at the very end of the powered descent since the spacecraft flies a bot beyond the landing site and returns. Therefore beacons along North-West to South-East direction are more sensitive to the downrange, while the ones perpendicular, i.e. along North-East to South-West are more sensitive to the crossrange.

4.3 One Beacon with Range and Range-rate Measurements (Case B)

The results for the single beacon configuration without the bearing measurement are now presented. The same 121 points in the grid in figure 10(a) have been used in this analysis. For each of them 100 Monte Carlo runs have been executed. Thus again 12,100 simulations have been done. The resulting filled contours can be seen in figures 17 to 19 .

The first result is, that the performance is generally improved with respect to the baseline setup. From a geometrical point of view, it was expected to see that the crossrange determination is worse for beacons along the landing groundtrack. The clear trends detectable in figures $17(\mathrm{~b}), 18(\mathrm{~b})$ and 19(b) are therefore consistent with the expectation. The more distant (perpendicular) to the ground-track, the more the crossrange is improved. In particular, when the beacon is along the groundtrack, the values of the evaluation functions under discussion are similar to the baseline. These performances are quite different from what happens in presence of the bearing, which helps the crossrange accuracy also when beacon is placed along the groundtrack as seen in the previous section.

From a geometrical point of view it was expected to see the opposite behavior for the downrange, i.e. a stronger positive impact on the downrange position error for beacons along the downrange. This is only the case for the phase when the lander is approaching high gate (see figure 18(a)). During the last phase of the landing (figure 19(a)) the trend is instead analogous to the one in crossrange. This is probably due the fact that the lander is passing over beacons along the groundtrack which is not the best configuration to observe downrange. However, the peak error is smaller than for the baseline. The fact that also figure 17 (a) shows this trend does not then mean that what said regarding the downrange observation is not verified, but this result is given to the large weight $J_{3, r}^{D R}$ peak values have on $J_{r}^{\text {tot }}$.

It is worth then noticing that the evaluation functions for the altitude component of the cases with and without the bearing mesurement are similar. It seems therefore clear that the bearing update does not influence much the determination of the altitude. 
It is clearly now confirmed, looking at figure $19(\mathrm{c})$, that best geometric configuration for altitude determination through range measurement is when beacon is placed at the landing site. A circular pattern is detectable in this figure (as it was also in figure 15(c)) with the minimum in the center which is located where the lander finds itself during the last phase of landing when it is descending almost vertically.
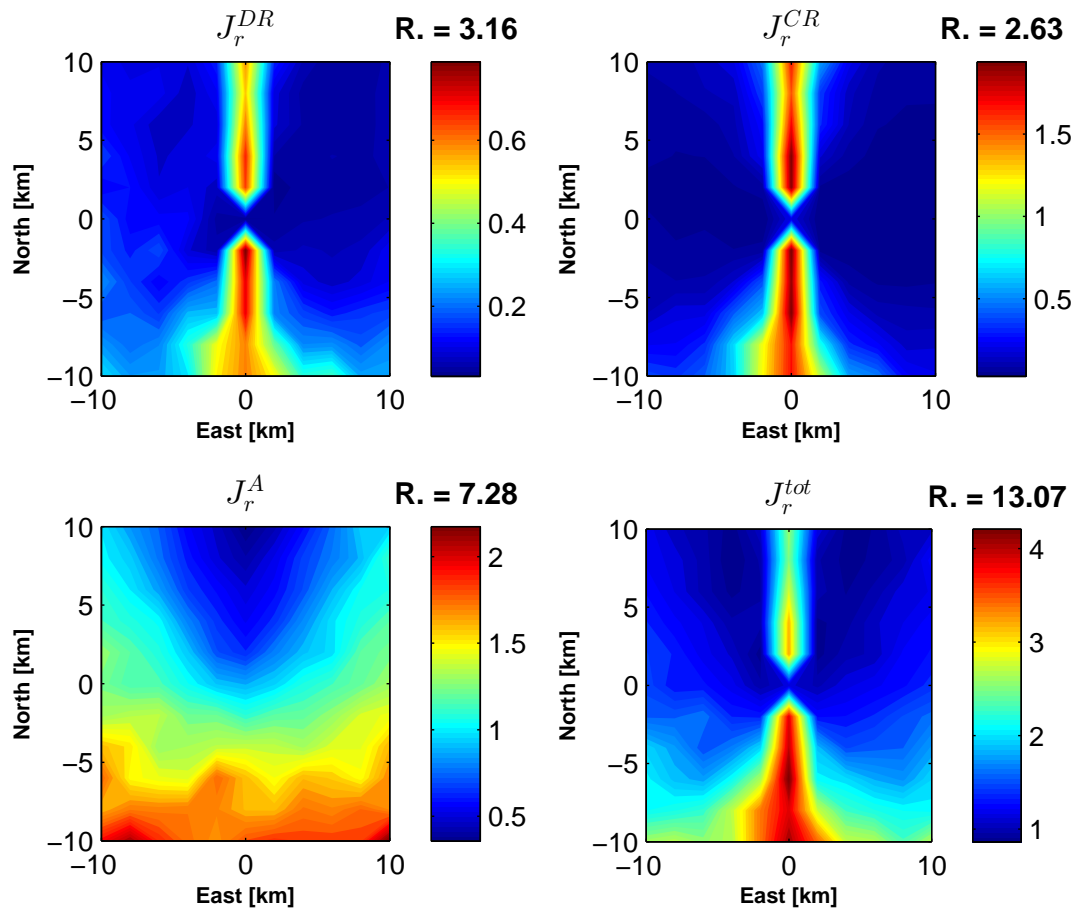

Fig. $17 J_{r}$ evaluation functions (case B). The value above each color bar shows the same cost function value for the baseline case without RF measurements.

It seems therefore that the impact of a beacon on navigation performances is positive, even without the measurement of the bearing. In particular, the position error is improved wherever the beacon is located inside the grid. Comparing the cases with and without bearing measurement it can be summarized:

- looking throughout figures 17 to 19 it is possible to see that the best area in which to maximize the performance is no more up-range along the groundtrack, but it seems that up-range and a little cross-track is the most favorable position;

- position related evaluation functions are overall larger, especially in the last phase of landing (see figure 15 and 19), in which the bearing is more effective; 

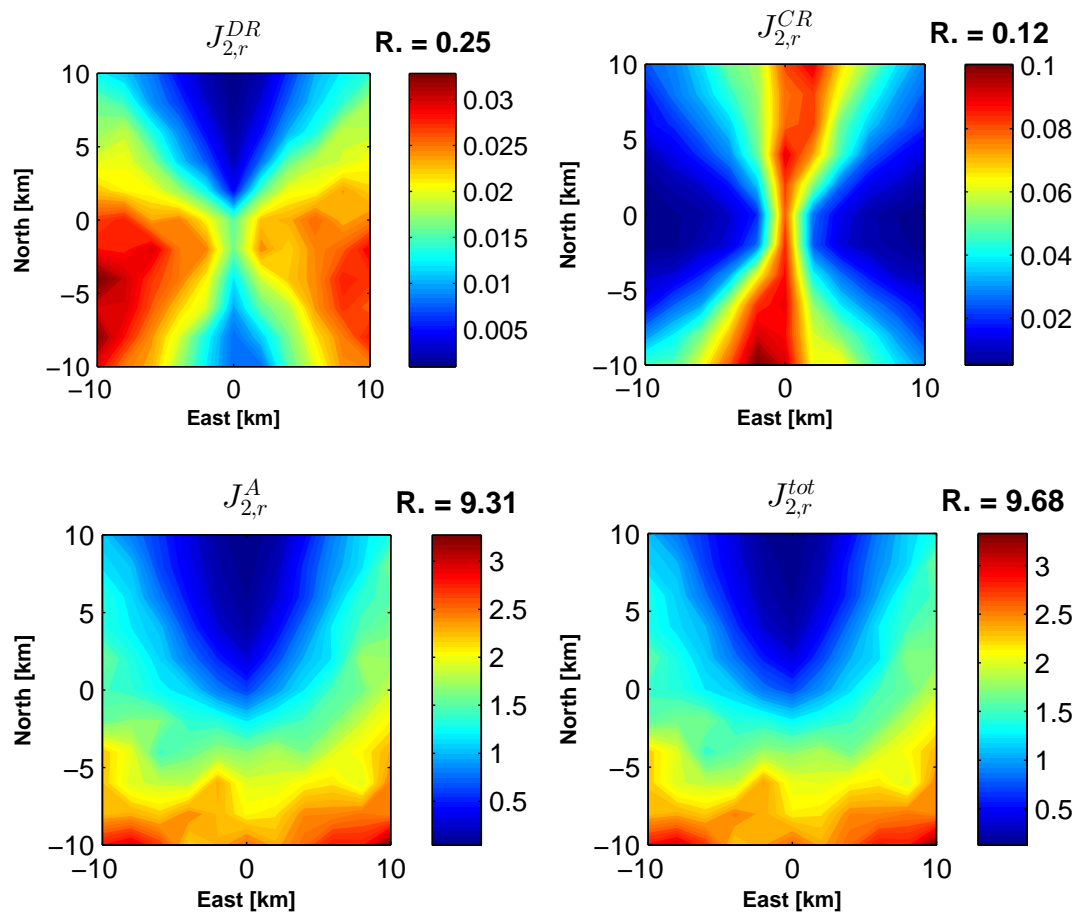

Fig. $18 J_{2, r}$ evaluation functions (case B). The value above each color bar shows the same cost function value for the baseline case without $\mathrm{RF}$ measurements.

- velocity related evaluation functions have substantially similar trends and values;

- the altitude position error, although decreasing a little with bearing enabled, is not much affected by it.

However, although it is clear that adding the bearing is better for the reduction of the errors, the single beacon configuration providing only range and range-rate measurements is already helping significantly the on-board baseline navigation system.

\subsection{Discussion of Results}

As it was discussed in the previous subsections the best navigation solution is achieved if the beacon is located close to the landing site with a distance in cross range to improve observability in case no bearing measurement is available. Together with the constraints discussed in section 3.5 a beacon location at a position $3000 \mathrm{~m}$ East and $1000 \mathrm{~m}$ North of the landing site is chosen (see figure 20). This position is a good trade-off between minimization of evaluation functions and final errors and the constraints imposed by the avoidance zone. 

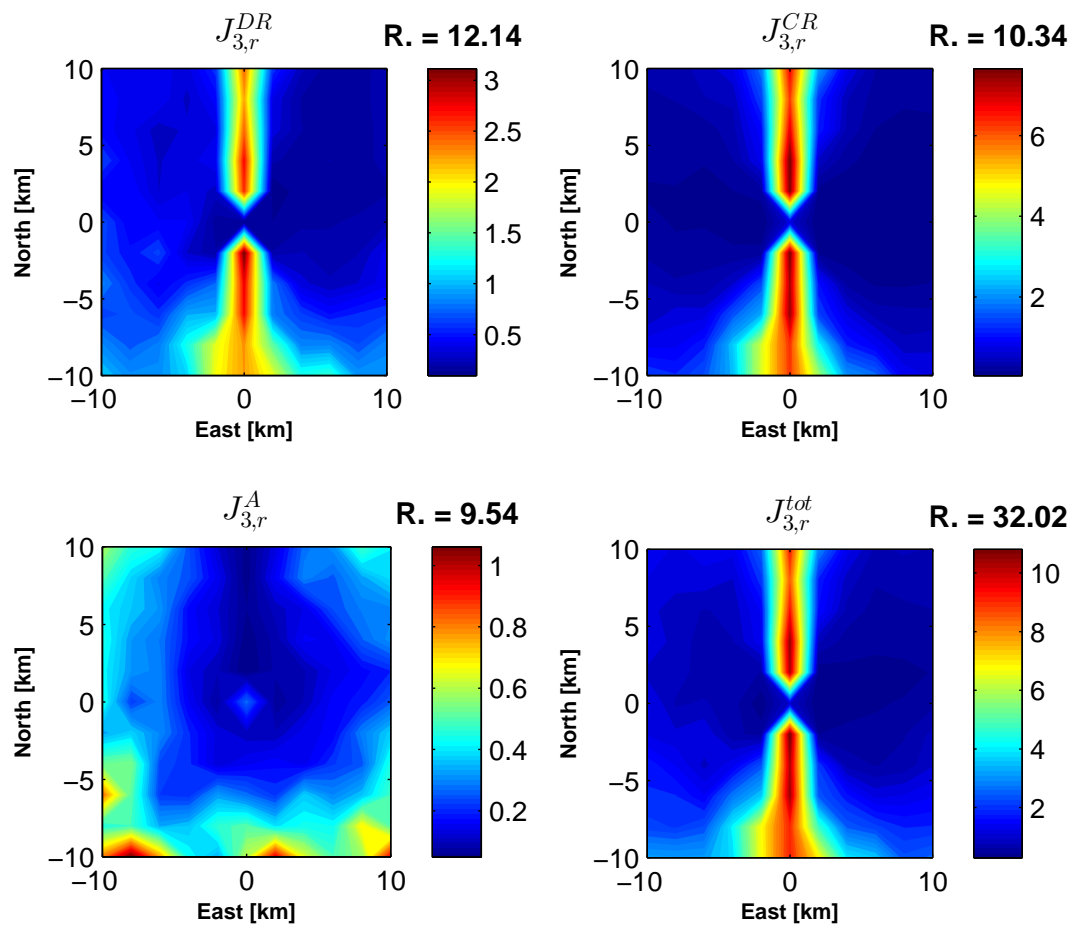

Fig. $19 J_{3, r}$ evaluation functions (case B). The value above each color bar shows the same cost function value for the baseline case without $\mathrm{RF}$ measurements.

Although in $[6,7]$ the avoidance zones were not considered, a similar optimal position for the beacon was found by covariance analysis.

For this beacon location the navigation results of both cases with RF measurements are compared to the baseline solution. In figures 21 and 22 the worst case errors for position and velocity in both cases with beacon measurements are shown as for the baseline navigation solution in figure 12 .

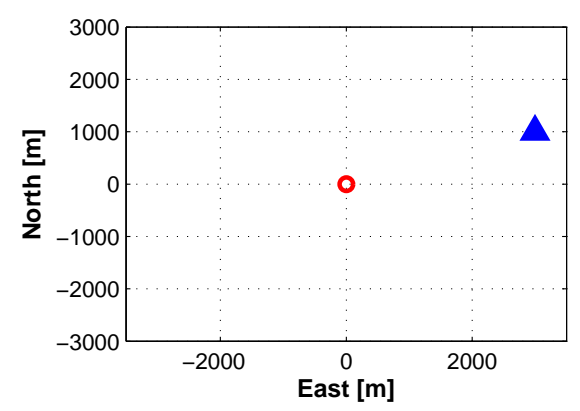

Fig. 20 Selected position of the single beacon (triangle) with respect to landing site (circle) 
Table 6 Performance of analyzed cases with respect to the baseline

\begin{tabular}{|c|c|c|c|c|c|c|c|}
\hline Parameter & Baseline & A & B & Parameter & Baseline & A & B \\
\hline$J_{r}^{D R}$ & 3.16 & 0.04 & 0.05 & $J_{v}^{D R}$ & 2.73 & 0.35 & 0.33 \\
\hline$J_{r}^{C R}$ & 2.73 & 0.03 & 0.03 & $J_{v}^{C R}$ & 0.27 & 0.23 & 0.23 \\
\hline$J_{r}^{A}$ & 7.28 & 0.95 & 0.96 & $J_{v}^{A}$ & 1.06 & 0.41 & 0.43 \\
\hline$J_{1, r}^{D R}$ & 0.09 & $<0.01$ & $<0.01$ & $J_{1, v}^{D R}$ & 6.61 & 0.48 & 0.47 \\
\hline$J_{1, r}^{C R}$ & 0.01 & 0.01 & 0.02 & $J_{1, v}^{C R}$ & 0.51 & 0.45 & 0.45 \\
\hline$J_{1, r}^{A}$ & 3.77 & 1.48 & 1.47 & $J_{1, v}^{A}$ & 2.43 & 0.61 & 0.67 \\
\hline$J_{2, r}^{D R}$ & 0.25 & 0.02 & 0.02 & $J_{2, v}^{D R}$ & 0.56 & 0.37 & 0.33 \\
\hline$J_{2, r}^{C R}$ & 0.12 & 0.02 & 0.03 & $J_{2, v}^{C R}$ & 0.13 & 0.12 & 0.12 \\
\hline$J_{2, r}^{A}$ & 9.30 & 1.00 & 1.02 & $J_{2, v}^{A}$ & 0.08 & 0.22 & 0.23 \\
\hline$J_{3, r}^{D R}$ & 12.14 & 0.11 & 0.15 & $J_{3, v}^{D R}$ & 0.13 & 0.13 & 0.12 \\
\hline$J_{3, r}^{C R}$ & 10.34 & 0.06 & 0.08 & $J_{3, v}^{C R}$ & 0.09 & 0.07 & 0.06 \\
\hline \multirow[t]{5}{*}{$J_{3, r}^{A}$} & 9.54 & 0.08 & 0.10 & $J_{3, v}^{A}$ & 0.26 & 0.39 & 0.38 \\
\hline & \multicolumn{2}{|c|}{ Parameter } & Baseline & $\mathrm{A}$ & B & & \\
\hline & \multicolumn{2}{|c|}{$\Delta E_{r, f}^{D R}[\mathrm{~m}]$} & 91.1 & 11.41 & 12.82 & & \\
\hline & \multicolumn{2}{|c|}{$\Delta E_{r, f}^{C R}[\mathrm{~m}]$} & 83.9 & 5.45 & 6.99 & & \\
\hline & \multicolumn{2}{|c|}{$\Delta E_{r, f}^{A}[\mathrm{~m}]$} & 0.23 & 0.31 & 0.31 & & \\
\hline
\end{tabular}

Although there are no RF measurement updates for the baseline navigation architecture, it is necessary to define a $t_{V I S}$ in order to use the equation 40 for $J, J_{1}$ and $J_{2}$. Therefore it is set to the value that it would have for a beacon placed exactly in the landing site. This choice is related to the fact that the landing site is in the center of the grid that was defined to study the single beacon configuration. Therefore the value of $t_{V I S}$ available there is kind of an average.

The evaluation function values and the worst case final errors for all cases are listed in table 6 . Values around unity or lower mean that the related worst case error is in the order of the requirement or below.

From the values for the baseline navigation solution in table 6 it can be seen that during powered descent the performance is getting worse. That means that especially for the position the navigation accuracy is not improving as much as required. The opposite happens for the velocities. They improve with time always meeting the requirements

From figures 12, 21 and 22, and from table 6 it is possible to see the impact of adding the updates from the RF measurement on the navigation accuracy during the second part of the powered descent:

- the most noticeable is the effect on the downrange errors, which suddenly diminish as the lander gets visible from the beacon (figure 21(a) and 21(b));

- the crossrange error component at the beginning seems not to be influenced by the presence of the beacon. But it starts decreasing following more or 
less the profile of the altitude error. This behavior is expected since the crossrange improvement is mostly given to the presence of the bearing;

- the errors in velocity seem to decrease with similar trend, but they look similar to the ones obtained without the use of the beacon;

- it is finally worth noticing the small increase in the position downrange and crossrange errors that starts $30-40$ seconds before the landing which is correlated to the exit from the visibility window.
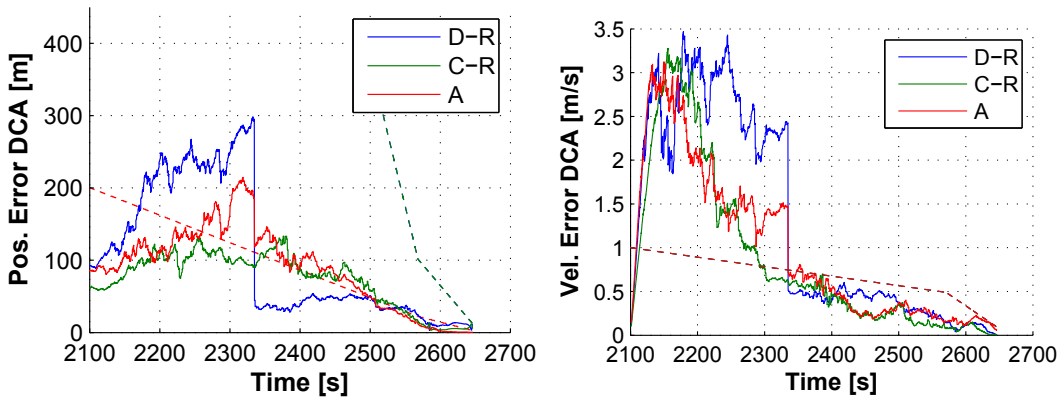

Fig. 21 Position and velocity error with $\mathrm{RF}$ range, range-rate and bearing measurements (case A). The dashed lines represent the requirements from table 4 and figure 9 .
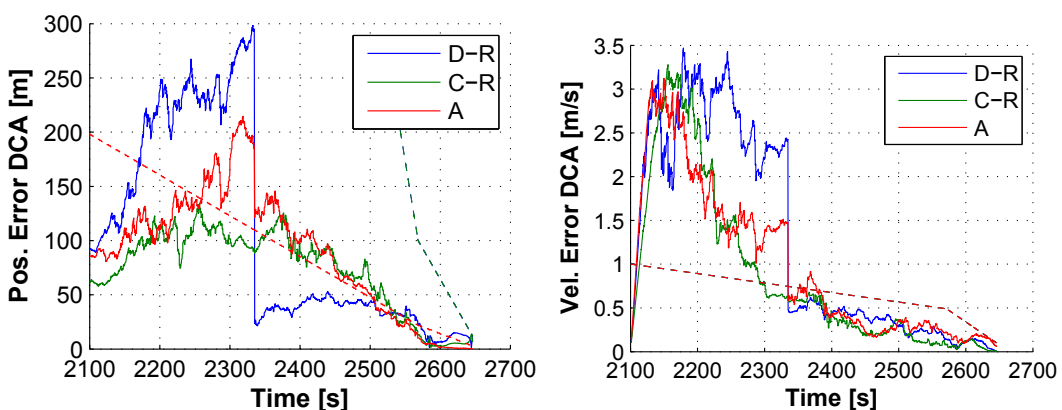

Fig. 22 Position and velocity error with RF range and range-rate measurements (case B). The dashed lines represent the requirements from table 4 and figure 9 .

If the worst case navigation errors for cases with the RF measurements (with and without bearing measurements see figures 21 and 22, and table 6) are compared it is hardly possible to see the slight improvement in position error as a result of the bearing measurements. Apparently, for a beacon located in the chosen position the performance with and without bearing is very similar. This is consistent with the fact that minimums of evaluation functions for both cases have been considered when choosing the location of the bearing. 


\section{Conclusions}

This paper presented a study on how the navigation for a lunar lander can be improved by using RF measurements from ground-based infrastructure (beacons). For that purpose the navigaton filter of an existing navigation system based on IMU, image processing and laser altimeter was augmented to process range, range-rate and bearing measurements from a received beacon signal. With this setup an extensive Monte-Carlo simulation campaign was carried out in order to analyse the impact of the RF measurements and of the position of the beacon on the navigation solution. Three cases were analysed: 1) the baseline case without RF measurements, 2) the support by range, rangerate and bearing measurement of a single beacon, and 3) the support by only range and range-rate measurements from a single beacon.

For comparing the results of the different setups and beacon locations evaluation functions have been defined which related the worst case error of the Monte-Carlo analysis to the requirements. For these evaluation functions contour plots were created which show the dependency of the different evaluation functions on the position of the beacon for the two different cases with beacon measurements.

For the baseline configuration of the navigation system the results indicate that the requirements for positioning are not met. The performance especially in the last phase of the landing is far beyond the required accuracy. This supports the need to improve the navigation with beacons.

The results for the cases with beacons show that independent of the location of the beacon in the analyzed grid the navigation performance for the position is much improved. Depending on the position the requirements can be met. So the choice of the beacon location has an impact especially on the last phase of the landing.

When comparing the two cases with RF measurements it can be seen that for the selected beacon position the impact of the additional bearing measurement is small. However, there are differences for other positions of the beacon. Especially for beacon positions along the groundtrack of the landing trajectory the position accuracy is worse if the beacon does not provide a bearing measurement. Thus it becomes important to consider possible approach directions before placing a beacon. From this fact one direction for further analyses can be derived: Find the best beacon position for a range of possible landing trajectories. This could be even extended if a beacon shall be placed for multiple landing sites in the vicinity.

Similarly, an interesting question to be investigated is the impact of more than one beacon on the navigation performance. Furthermore, the impact of deviation from the nominal trajectory due to control errors on the navigation performance should be also analyzed.

Another direction for future work is augmenting the navigation filter to estimate the clock error and clock drift of the onboard receiver and considering the positioning error of the beacon. In the current analysis the range and rangerate measurements were used with very conservative error values to account for 
the unknown clock error, clock drift and beacon position. A next step would analyze the impact of these unknowns on the navigation performance.

\section{References}

1. Andert, F., Ammann, N., Maass, B.: Lidar-aided camera feature tracking and visual slam for spacecraft low-orbit navigation and planetary landing. In: CEAS EuroGNC 2015 (2015). URL http://elib.dlr.de/96323/

2. Batista, P., Silvestre, C., Oliveira, P.: Single beacon navigation: Observability analysis and filter design. In: American Control Conference (ACC), 2010, pp. 6191-6196 (2010). DOI 10.1109/ACC.2010.5531613

3. Beggins, A.J., Canney, L.M., Dolezal, A.B., States., U.: Conceptual development of a ground-based radio-beacon navigation system for use on the surface of the moon [microform] / prepared by Andrew J. Beggins, Lora M. Canney, Anna Belle Dolezal. Mechanical Engineering Design Project, University of Texas at Austin Austin, Tex (1988)

4. Bora, L.: Ground beacons to enhance lunar landing autonomous navigation architectures. Master's thesis, Politecnico di Milano (2015). URL http://elib.dlr.de/100498/

5. Chelmins, D.T., Welch, B.W., Sands, O.S., Nguyen, B.V.: A kalman approach to lunar surface navigation using radiometric and inertial measurements. Tech. Rep. 20090027870, National Aeronautics and Space Administration, NASA Glenn Research Center; Cleveland, OH, United States (2009). URL http://ntrs.nasa.gov/search.jsp?R=20090027870

6. Christensen, D., Geller, D.: Terrain-relative and beacon-relative navigation for lunar powered descent and landing. The Journal of the Astronautical Sciences 58(1), 121151 (2011). DOI 10.1007/BF03321162. URL http://dx.doi.org/10.1007/BF03321162

7. Christensen, D.P.: Terrain-relative and beacon-relative navigation for lunar powered descent and landing. Master's thesis, Utah State University, Logan, Utah (2009). URL http://digitalcommons.usu.edu/cgi/viewcontent.cgi? article=1257\&context=etd

8. Davies, J.L., Striepe, S.A.: Advances in POST2 End-to-End Descent and Landing Simulation for the ALHAT Project. AIAA-2008-6938. American Institute of Aeronautics and Astronautics (2008)

9. Department of Defence and Department of Transport: 2001 Federal Radionavigation Systems. National Technical Information Service, Springfield VA 22161 (2001)

10. Durrant-Whyte, H., Bailey, T.: Simultaneous Localization and Mapping: Part I. IEEE Robotics \& Automation Magazine pp. 99-108 (2006)

11. Epp, C., Smith, T., NASA, H.: Autonomous Precision Landing and Hazard Detection and Avoidance Technology (ALHAT). In: 2007 IEEE Aerospace Conference, pp. 1-7 (2007)

12. Grelier, T., Guidotti, P.Y., Delpech, M., Harr, J., Thevenet, J.B., Leyre, X.: Formation flying radio frequency instrument: First flight results from the prisma mission. In: 2010 5th ESA Workshop on Satellite Navigation Technologies and European Workshop on GNSS Signals and Signal Processing (NAVITEC), pp. 1-8 (2010). DOI 10.1109/NAVITEC.2010.5708059

13. Heise, D.T.S.G., Steffes, S.R., Theil, S.: Filter design for small integrated navigator for planetary exploration. In: 61. Deutscher Luft- und Raumfahrtkongress 2012 (2012). URL http://elib.dlr.de/81142/

14. Houdou, B., the ESA NEXT Lunar Lander Team: NEXT Lunar Lander with In-Situ Science and Mobility: Phase A Mission Study, Mission Requirements Document. Internal Report NEXT-LL-MRD-ESA(HME)-0001, ESA (2008)

15. Huang, Y., Hu, X., Li, P., Cao, J., Jiang, D., Zheng, W., Fan, M.: Precise positioning of the chang'e-3 lunar lander using a kinematic statistical method. Chinese Science Bulletin 57(35), 4545-4551 (2012). DOI 10.1007/s11434-012-5484-5. URL http://dx.doi.org/10.1007/s11434-012-5484-5

16. Kayton, M., Fried, W.R.: Avionics Navigation Systems, 2nd edn. John Willey and Sons Inc. (1997) 
17. Lockner, E., Oehlschlägel, T., Theil, S., Knauer, M., Tietjen, J., Büskens, C.: Real-time capable trajectory synthesis via multivariate interpolation methods for a moon landing manoeuvre. CEAS Space Journal Vol 6(2), 107-118 (2014). URL http://elib.dlr.de/97751/

18. Maass, B., et. al.: An Edge-Free, Scale-, Pose- and Illumination-Invariant Approach to Crater Detection for Spacecraft Navigation. In: 7th International Symposium on Image and Signal Processing and Analysis (ISPA 2011). Dubrovnik, Croatia (2011)

19. Montenbruck, O., Ebinuma, T., Lightsey, E., Leung, S.: A real-time kinematic gps sensor for spacecraft relative navigation. Aerospace Science and Technology 6(6), 435-449 (2002). URL http://elib.dlr.de/11383/. LIDO-Berichtsjahr=2002,

20. NASA: How to protect and preserve the historic and scientific value of u.s. government lunar artifacts. Tech. rep., National Aeronautics and Space Administration (2011)

21. Oehlschlägel, T., Theil, S., Krüger, H., Knauer, M., Tietjen, J., Büskens, C.: Optimal Guidance and Control of Lunar Landers with Non-throttable Main Engine. Advances in Aerospace Guidance, Navigation and Control (2011)

22. Parkinson, B., Spilker Jr., J.: Global Positioning System: Theory and Applications Volume II. Progress in Astronautics and Aeronautics, Vol. 164. AIAA (1996)

23. Schier, J.: Nasa's lunar space communication and navigation architecture. In: International Communications Satellite Systems Conferences (ICSSC), pp. -. American Institute of Aeronautics and Astronautics (2008). DOI 10.2514/6.2008-5476. URL http://dx.doi.org/10.2514/6.2008-5476

24. Steffes, S.R.: Development and analysis of shefex-2 hybrid navigation system experiment. Ph.D. thesis, DLR Bremen (2013). URL http://elib.dlr.de/82946/

25. Steffes, S.R., Theil, S., Dumke, M., Heise, D., Sagliano, M., Samaan, M.A., Laan, E., Durkut, M., Duivenvoorde, T., Nijkerk, D., Schulte, J., Söderholm, S., Skaborn, D., Berkhout, J., Esposito, M., Conticello, S., Visee, R., Monna, B., Stelwagen, F.: SINPLEX: a Small Integrated Navigation System for Planetary Exploration. In: 36th Annual AAS Guidance and Control Conference. AAS, Breckenridge, Colorado (2013). AAS 13-043

26. Theil, S., Krüger, H.: Analyse Missionen. Internal Report AT-RYNR-TN-002, DLR (2010)

27. Wedler, A., Hellerer, M., Rebele, B., Gmeiner, H., Vodermayer, B., Bellmann, T., Barthelmes, S., Rosta, R., Lange, C., Witte, L., Schmitz, N., Knapmeyer, M., Czeluschke, A., Thomsen, L., Waldmann, C., Flögel, S., Wilde, M., Takei, Y.: Robex ? components and methods for the planetary exploration demonstration mission. In: 13th Symposium on Advanced Space Technologies in Robotics and Automation (ASTRA), ASTRA. ESAWebsite (2015). URL http://elib.dlr.de/98242/ 